\title{
Phenomenological model of weakly damped Faraday waves and the associated mean flow
}

\author{
José M. Vega \\ E.T.S.I. Aeronáuticos, Lniversidad Politécnica de Madrid. Plaza Cardenal Cisneros 3, 28040 Madrid, Spain \\ Sten Rüdiger and Jorge Viñals \\ School of Computational Science and Information Technology, Florida State University, Tallahassee. Florida 32306-4120, USA
}

\begin{abstract}
A phenomenological model of parametric surface waves (laraday waves) is introduced in the limit of small viscous dissipation that accounts for the coupling between surface motion and slowly varying streaming and large-scale flows (mean flow). The primary bifurcation of the model is to a set of standing waves (stripes, given the functional form of the model nonlinearities chosen here). Our results for the secondary instabilities of the primary wave show that the mean flow leads to a weak destabilization of the base state against Eckhaus and transverse amplitude modulation instabilities, and introduces a longitudinal oscillatory instability which is absent without the coupling. We compare our results with recent one-dimensional amplitude equations for this system systematically derived from the governing hydrodynamic equations.
\end{abstract}

DOI: 10.1103/ThysRevE.70.046306 PACS number(s): 47.54.+r, 47.20.-k, 47.35.+i, 47.32. $-\mathrm{y}$

\section{INTRODUCTION}

The purpose of this paper is to couple a phenomenological order parameter model of parametric surface waves in the limit of weak viscous dissipation to slowly varying llows (mean flows). To date, most theories of parametric surface waves near onset have neglected such flows despite the observation that their effect is of the same order as other cubic nonlinear conservative terms retained. The coupling to the phenomenological model presented here allows us to discuss the simplest consequences that these flows have in a laterally unbounded geometry, namely, shifts in thresholds of secondary instabilities of the base pattern of standing waves, and the appearance of a new longitudinal oscillatory instability.

When a layer of an incompressible fluid is vibrated periodically along the direction normal to the free surface at rest, it can cxhibil parametrically driven surface waves, also known as Faraday waves [1-4]. Just above the primary instability of the planar free surface, a set of standing surface waves emerges leading to a stationary pattern with a symmetry that depends on the physical parameters of the fluid and the frequency of the forcing [5-8]. Intricate phenomena appear in the limit of weak viscous dissipation in which nonlinear wave interactions responsible for wave saturation and pattern selection are dominated by triad resonant interactions [9-11]. Whereas the first bifurcation away from planarity is to a set of standing waves in which mean flow effects are absent, mean flows are expected to be important in determining the stability of the primary waves, and more generally in weakly damped systems. In this latter case, standing-wave amplitude equations can be expected to be valid only very close to onset.

Current weakly nonlinear theory is restricted to the small region above threshold in which standing waves are stable, a slate in which mean flows vanish identically. However, the contribution from mean flows to the equations governing the slow cvolution of the surface waves can be of the same order as the standard cubic nonlinear and conservative terms which are always retained. Thus weakly nonlinear corrections to surface waves and mean flows must be considered simultaneously, which has not been done in three dimensions in the limit considered below (see [12] for an analysis of this limit in two dimensions and [13-15] for an analysis of related limits). The effects considered here are not unlike other known phenomenology that includes the streaming flow produced by fixed surface waves ([16-19], and references therein) and the evolution of surface waves in the presence of a fixed vortical flow $[20,21]$.

A consistent introduction of mean flow effects into the amplitude equations for Faraday waves requires explicit consideration of special limits that involve the physical dimensions of the container. We specifically focus here on the case of a fluid depth that is logarithmically large compared to the wavelength, and derive a set of evolution equations for the surface waves and the associated mean flow in the double limit of small viscosity and large aspect ratio (the ratio between the lateral size of the container and the wavelenglh). We find two separate contributions to mean flow, namely an inviscid contribution arising from the slowly varying motion of the free surface, similar to the one appearing in classical Davey-Stewartson models [22], and a viscous one resulting from a slowly varying shear stress produced by the oscillatory boundary layer attached to the free surface. This latter contribution describes vorticity transport (by viscous diffusion or convection) from the boundary layer into the bulk [23].

An important simplification in our analysis is that the cubic nonlinear terms of the phenomenological model are chosen so as to lead to a stripe pattern above onset instead of a square pattern as experimentally observed in the limit of weak viscous dissipation. While it is a simple matter to modify the functional form of the cubic term to produce square patterns, we have chosen to first clarify the effect of mean flows on slow modulations of a stripe pattern. There is no satisfactory theory at present that can account for the interaction between slow spatial modulation of the waves 
and mean flows in three dimensions, and the case of stripes is considerably simpler than other symmetries involving a larger number of plane-wave components at onset.

\section{FORMULATION}

We consider a fluid layer of unperturbed depth $d$ supported by a horizontal plate that is vibrating vertically with an amplitude $a^{*}$ and a frequency $2 \omega^{*}$, where the superscript * denotes dimensional quantities. In order to facilitate comparison with related results in $[11,24]$, we use for adimensionalization the characteristic time $\omega^{*-1}$ and length $k^{k+1}$, where the wave number $k^{*}$ is related to $\omega^{\prime \prime}$ by the inviscid dispersion relation

$$
\omega^{* 2}=g^{*} k^{*}+\sigma^{*} k^{* 3} / \rho^{*},
$$

in terms of the gravitational acceleration $g^{\prime \prime}$, the surface tension $\sigma^{*}$, and the density $\rho^{*}$, all assumed constant. Here we are assuming that the wavelength $k^{* 1}$ is (at least somewhat) small compared with the depth of the container. The resulting dimensionless continuity and Navier-Stokes equations in a reference frame attached to the vibrating container, with the $z=0$ plane at the unperturbed free surface, are

$$
\boldsymbol{\nabla} \cdot \boldsymbol{u}+\partial_{z} w=0,
$$

$$
\partial_{t} \boldsymbol{u}-w\left(\boldsymbol{\nabla} w-\partial_{z} \boldsymbol{u}\right)-\boldsymbol{u}^{\prime} \boldsymbol{\nabla} \cdot \boldsymbol{u}^{\prime}=-\boldsymbol{\nabla} p+\gamma\left(\boldsymbol{\nabla}^{2} \boldsymbol{u}+\partial_{z z}^{2} \boldsymbol{u}\right) / 2,
$$

$$
\partial_{l} w+\boldsymbol{u} \cdot\left(\boldsymbol{\nabla} w-\partial_{z} \boldsymbol{u}\right)=-\partial_{z} p+\gamma\left(\nabla^{2} w+\partial_{z z}^{2} w\right) / 2,
$$

in $-d<z<h(x, y, t)$, with boundary conditions resulting from no slip at the supporting plate,

$$
\boldsymbol{u}=0, \quad w=0 \text { at } z=-d,
$$

and kinematic compatibility and equilibrium of tangential and normal stresses at the free surface,

$$
\begin{gathered}
\partial_{t} h+\boldsymbol{u} \cdot \boldsymbol{\nabla} h=w, \\
\partial_{z} \boldsymbol{u}+\boldsymbol{\nabla} w-\left(\boldsymbol{\nabla} \boldsymbol{u}+\boldsymbol{\nabla} \boldsymbol{u}^{\top}\right) \cdot \boldsymbol{\nabla} h \\
+\left[2 \partial_{z} w-\left(\partial_{z} \boldsymbol{u}+\boldsymbol{\nabla} w\right) \cdot \boldsymbol{\nabla} h\right] \boldsymbol{\nabla} h=0, \\
p-\left(|\boldsymbol{u}|^{2}+w^{2}\right) / 2-[4 a \sin 2 t+1-\Gamma] h \\
+\boldsymbol{\Gamma} \nabla \cdot\left[\boldsymbol{\nabla} h /\left(1+|\nabla h|^{2}\right)^{1 / 2}\right] \\
=\gamma\left\{\partial_{z} w-\left(\partial_{z} \boldsymbol{u}+\boldsymbol{\nabla} w\right) \cdot \nabla h\right. \\
\left.+\left[\boldsymbol{\nabla} h \cdot\left(\boldsymbol{\nabla} \boldsymbol{u}+\boldsymbol{\nabla} \boldsymbol{u}^{\top}\right) / 2\right] \cdot \boldsymbol{\nabla} h\right\} /\left(1+|\boldsymbol{\nabla} h|^{2}\right)
\end{gathered}
$$

at $z=h$. Here

$$
\boldsymbol{u}=(u, v, 0)
$$

and $w$ are the horizontal and vertical velocity components,

$$
\boldsymbol{\nabla}=\left(\partial_{x}, \partial_{y}, 0\right)
$$

denotes the horizontal gradient, the superscript $\perp$ over a horizontal vector denotes the result of rotating the vector $90^{\circ}$ counterclockwise, namely,

$$
\boldsymbol{u}^{-}=(-v, u, 0),
$$

and the superscript $T$ over a tensor denotes the transpose; $p\left(=(\right.$ pressure $\left.\left.)+\left(|\boldsymbol{u}|^{2}+w^{2}\right) / 2+[1-\Gamma+4 a \sin (2 t)] z\right)\right)$ is aconveniently modified pressure, and $h$ is the (vertical) free surface deflection. For simplicity, we do not consider lateral walls, but impose periodic boundary conditions in two horizontal directions, namcly

$$
\begin{gathered}
(\boldsymbol{u}, w, p)\left(x+L_{1, y, z, t)}=(\boldsymbol{u}, w, p)\left(x, y+L_{2}, z, t\right)\right. \\
=(\boldsymbol{u}, w, p)(x, y, z, t), \\
f\left(x+l_{1}, y, t\right)=f\left(x, y+l_{2}, t\right)=f(x, y, t) .
\end{gathered}
$$

And for convenience we also consider the vertically integrated continuity equation

$$
\partial_{i} h+\nabla \cdot\left(\int_{-d}^{h} \boldsymbol{u} d z\right)=0
$$

oblained upon integration of Eq. (2) in $-d<z<h$ and substitution of Eq. (6).

\section{A. Multiple scale analysis: Oscillatory and mean flows}

We consider next a specific range of parameters in which it is possible to simplify the problem by separating fluid motion into a "Tast" oscillatory component and a "slow" mean llow. In particular, we consider the system of surface waves near onset, and in the limits of a very large lateral surface and weak viscous dissipation. The problem depends on the following dimensionless parameters: the dimensionless viscosity $\gamma=2 \nu^{*} k^{* 2} / \omega^{*}$ (with $\nu^{*}$ the kincmatic viscosity), the gravity-capillary contribution $\mathbf{I}=\sigma^{*} k^{* 3} /\left(\rho^{*} \omega^{* 2}\right)$, the forcing amplitude $a=a^{\prime \prime} k^{\prime \prime}$, the container depth $d=d^{\prime \prime} k^{*}$, and the aspect ratios $L_{1}$ and $L_{2}$; note that, according to Eq. (1), 0 $\leqslant \Gamma \leqslant 1$ and the extreme cases $\Gamma=0$ and 1 correspond to the purely gravitational and purely capillary limits, respectively. The approximation below requires (a) that the aspect ratio of the container be large, (b) that the surface waves be weakly damped, (c) that a small wavelength be exhibited compared to the container's depth, and (d) a small stecpness, which in turn require that

$$
L \gg 1, \quad d \gg 1, \quad \gamma \ll 1, \quad|\nabla h| \ll 1, \quad a \ll 1,
$$

where $I_{2} \leqslant \min \left\{I_{1}, I_{2}\right\}$. The large spatial scale set by the (large) aspect ratio introduces a slow horizontal scale over which both spatial and temporal modulations are expected to occur. As suggested by the 2D case [12], this scale is expected to be determined (in the equations for the oscillatory flow associated with the surface waves) by the balance between cubic nonlinearity and either (i) transport with the group velocity or (ii) dispersion. And $d$ must be not 100 large; sec below. For the sake of clarity, we assume that $d$ is logarithmically large compared to the remaining small parameters (namcly $\gamma, a$, and $l,{ }^{1}$ ) and we shall treat $d$ as an O(1) parameter. In fact, for simplicity we consider the distinguished limit 


$$
\gamma^{2} \leqslant e^{-d} \ll L^{-1} \sim \gamma \sim a \sim \varepsilon^{2},
$$

where $\varepsilon$ is a measure of the surface-wave amplitude; sec Eq. (18) bclow. The estimates $\gamma \sim a \sim c^{2}$ result from imposing that lincar damping, cubic nonlincarity, and parametric forcing be of the same order. Therefore, we are implicitly assuming that the coefficient of the cubic term is $O(1)$, which excludes triad resonances [11]. If these are present, the cubic coefficient becomes $O\left(\gamma^{-1}\right)$ and a different scaling applies. In order to concentrate on the effects of mean flows, we exclude triad resonances in what follows.

Under these assumptions, we shall (implicitly) use a multiscale analysis in both (horizontal) space and time. But in order to make a not too technical presentation and to avoid obscuring the main idcas with a too involved notation, we shall use only one time variable and one space variable in each horizontal direction. The basic (fast) scales involve $O(1)$ increments of $x, y$, or $t$. A magnitude $\psi$ that exhibits these fast scales at leading order is such that

$$
|\partial \psi| \partial x|\sim| \psi|, \quad| \partial|f| \partial y|\sim| \psi \mid, \quad \text { or }|\partial| \psi f \partial t|\sim| \psi \mid,
$$

and it will be said to depend strongly on the associated variable $x, y$, or $t$. If instead the magnitude only changes over the slower scale at leading order, namely, if

$$
|\partial \psi / \partial x| \ll|\psi|, \quad|\partial \psi / \partial y| \ll|\psi|, \quad \text { or }|\partial \psi / \partial t| \ll|\psi|,
$$

then the magnitude will be said to depend weakly on the associated variable, $x, y$, or $t$. To proceed, we decompose the flow variables and the free surface deflection into oscillatory and time-averaged parts, associated with the surface waves and the mean llow (denoted hercafter with the superscripts $O$ and $m$ ), respectively, as

$$
(\boldsymbol{u}, w, p, h)=\varepsilon\left(\boldsymbol{u}^{o}, w^{\prime \prime}, p^{\prime \prime}, h^{\prime \prime}\right)+\varepsilon^{2}\left(\boldsymbol{u}^{m}, w^{m}, p^{m}, h^{m}\right),
$$

where (i) the (oscillatory) flow variables associated with the surface waves are required to be such that

$$
\left\langle\boldsymbol{u}^{o}\right\rangle^{t s}=0,\left\langle w^{o}\right\rangle^{t s}=\left\langle p^{o}\right\rangle^{t s}=\left\langle h^{o}\right\rangle^{t s}=0,
$$

with \langle\rangle$^{t s}$ standing here and hereafter for the time average in the basic oscillating period

$$
\langle\psi\rangle^{t s}=(2 \pi)^{l} \int_{1}^{1 / 2 \pi} \psi d t
$$

and (ii) the variables associated with the mean flow are required to depend weakly on time; more precisely, we assume that

$$
\begin{aligned}
\left|\partial_{t} \boldsymbol{u}^{m}\right| \sim \varepsilon^{2}\left|\boldsymbol{u}^{m}\right|, & \left|\partial_{t} w^{m}\right| \sim \varepsilon^{2}\left|w^{m}\right|, \quad\left|\partial_{t} p^{m}\right| \sim \varepsilon^{2}\left|p^{m}\right|, \\
& \left|\partial_{t} h^{m}\right| \sim \varepsilon^{2}\left|h^{m}\right|,
\end{aligned}
$$

where we are anticipating the time scale for the slow evolution of the mean flow, $t \sim \varepsilon^{-2}$. Also we anticipate that the rescaled flow variables $\boldsymbol{u}^{\prime \prime}, \ldots, h^{o}, \boldsymbol{u}^{m}, \ldots, h^{m}$ are at most of order unity; sec below. The mean flow is described in terms of the time-averaged velocity $\varepsilon^{2} \boldsymbol{u}^{m}$, which is the fulerian velocity and does not coincide in general with the velocity associated with the time average of the trajectories of fluid elements. The latter is the lagrangian mean velocity, or mass transport velocity (denoted here as $\varepsilon^{2} u^{m t}$ ), which is the appropriatc velocity to analy $\%$ mean trajectorics of passive scalars (sce, c.g., $[25,26]$ in conncction with chaotic advec(ion [27]). The difference between the two is the Stokes drift so that, in the notation of this paper, its horizontal and vertical components, scaled with $\varepsilon^{2}$, are given by [28]

$$
\begin{aligned}
& \boldsymbol{u}^{m t}-\boldsymbol{u}^{m}=\boldsymbol{u}^{s d}=\left\langle\left(\int^{t} \nabla \phi \cdot \nabla\right) \nabla \phi+\left(\int^{t} \partial_{z} \phi\right) \partial_{z} \nabla \phi\right\rangle^{t s}, \\
& w^{m t}-w^{m}=w^{s d}=\left\langle\left(\int^{t} \nabla \phi\right) \cdot \nabla\left(\partial_{z} \phi\right)+\left(\int^{t} \partial_{z} \phi\right) \partial_{z z} \phi\right\rangle^{t s},
\end{aligned}
$$

in a first approximation, where we are anticipating Eq. (25) below, and the operator $\int^{t}$ is defined as

$$
\int^{t} \psi=\left\langle\int^{t} \psi d t\right\rangle^{t o},
$$

with \langle\rangle$^{t(t)}$ standing hercafter for the time-oscillatory part, defined as

$$
\left.\langle\psi\rangle\rangle^{t o}=\psi\right\rangle-\langle\psi \mid \psi\rangle^{t s}
$$

By definition, Eq. (23) is independent of $t_{0}$.

\section{B. Oscillatory flow}

We begin by deriving the equations governing the oscillatory flow associated with the surface waves which exhibits a thin viscous boundary layer of $O(\sqrt{\gamma})$ thickness atlached to the free surface. In the bulk region outside this boundary layer, the oscillatory velocity components and the pressure are given by

$$
\begin{gathered}
\boldsymbol{u}^{o}=\boldsymbol{\nabla} \phi+\varepsilon^{2}\left[\left(\int^{t} \partial_{z} \phi\right)\left(\boldsymbol{\nabla} w^{m}-\partial_{z} \boldsymbol{u}^{m}\right)\right. \\
\left.+\left(\int^{t} \boldsymbol{\nabla} \phi\right)^{-} \boldsymbol{\nabla} \cdot \boldsymbol{u}^{m \perp}\right]^{t s}+O\left(\varepsilon^{3}\right), \\
w^{o}=\partial_{z} \phi-\varepsilon^{2}\left(\int^{t} \boldsymbol{\nabla} \phi\right) \cdot\left(\boldsymbol{\nabla} w^{m}-\partial_{z} \boldsymbol{u}^{m}\right)+O\left(\varepsilon^{3}\right), \\
p=-\partial_{t} \phi,
\end{gathered}
$$

as obtained upon substitution of Eq. (18) into Eqs. (3) and (4), where $\phi$ is the velocity potential. Thus the oscillatory flow is potential at leading order, but not at order $\varepsilon^{2}$, which must be retained in what follows. Substitution of Eq. (25) into Eq. (2) yields, after some algebra. 


$$
\begin{gathered}
\nabla^{2} \phi+\partial_{z z}^{2} \phi+\varepsilon^{2}\left(\int^{t} \partial_{z} \phi\right) \nabla \cdot\left(\nabla w^{m}-\partial_{z} \boldsymbol{u}^{m}\right) \\
+\varepsilon^{2} \boldsymbol{\nabla} \cdot\left[\left(\int^{t} \nabla \phi\right)^{\perp} \boldsymbol{\nabla} \cdot \boldsymbol{u}^{m-}\right] \\
-\varepsilon^{2}\left(\int^{t} \nabla \phi\right) \cdot \partial_{z}\left(\nabla w^{m}-\partial_{z} \boldsymbol{u}^{m}\right)=0 \\
\text { for }-d<z<0 .
\end{gathered}
$$

Here we are taking the upper boundary at the unperturbed free surface, which can be done because $h$ is small. The boundary conditions at the upper boundary must include the effect of the vortical flow in the boundary layer atlached to the frec surface on the oscillating llow in the bulk. To the approximation relevant here, this only requires us to replace the boundary condition (7) by (see, e.g.. [11])

$$
\partial_{t} h+\boldsymbol{u} \cdot \boldsymbol{\nabla} h=w+W(x, y, t) \text { al } z=h,
$$

where $W$ is given by

$$
\partial_{t} W(x, y, t)=\gamma \nabla^{2}\left(\partial_{z} \phi\right)
$$

this equation can be integrated to obtain

$$
W=\gamma \int^{t} \nabla^{2}\left(\partial_{I} \phi\right)
$$

Thus, to the approximation relevant here, Eq. (13) can be rewritten as

$$
\partial_{t} h+\nabla \cdot\left(\int_{-d}^{h} u d z\right)=\gamma \int \nabla^{2}\left(\partial_{z} \phi\right) .
$$

The boundary conditions for the oscillatory flow at the unperturbed free surface are now obtained by a Taylor expansion from Eqs. (8), (25), and (30), and are found to be

$$
\begin{aligned}
& \partial_{t} h^{o}-\partial_{z} \phi+\varepsilon^{2}\left(\int^{t} \boldsymbol{\nabla} \phi\right) \cdot\left(\boldsymbol{\nabla} w^{m}-\partial_{z} u^{m}\right)+c\left\langle\boldsymbol{\nabla} \cdot\left(h^{o} \boldsymbol{\nabla} \phi\right)\right\rangle^{t^{o}} \\
& +r^{2} \boldsymbol{\nabla} \cdot\left[h^{m} \boldsymbol{\nabla} \phi+h^{o} \boldsymbol{u}^{m}+\left(h^{o}\right)^{2} \partial_{z} \boldsymbol{\nabla} \phi / 2\right] \\
& =\gamma \int^{t} \nabla^{2}\left(\partial_{z} \phi\right) \\
& \partial_{t} \phi+\varepsilon\left\langle h^{\circ} \partial_{t z}^{2} \phi\right\rangle^{\dagger o}+\varepsilon^{2}\left[h^{m} \partial_{t z}^{2} \phi-h^{o} \partial_{s} p^{m}+\left(h^{o}\right)^{2} \partial_{t z z}^{3} \phi / 2\right] \\
& +\varepsilon\left\langle|\boldsymbol{\nabla} \phi|^{2}+\left|\partial_{z} \phi\right|^{2}\right\rangle^{t_{0}} / 2+\varepsilon^{2}\left[u^{m} \cdot \boldsymbol{\nabla} \phi+h^{\circ} \partial_{z}\left(|\boldsymbol{\nabla} \phi|^{2}\right.\right. \\
& \left.+\left|\partial_{z} \phi\right|^{2}\right) / 2+4 \alpha\left\langle h^{o} \sin 2 t\right\rangle^{\prime \prime}+(1-\Gamma) h^{\prime \prime} \\
& -\Gamma \nabla \cdot\left[\nabla h^{o} /\left(1+\varepsilon^{2}\left|\nabla h^{o}\right|^{2}\right)^{1 / 2}\right]+\gamma \partial_{z z}^{2} \phi=2,
\end{aligned}
$$

where we are using Eq. (58) below. The boundary condition (5) at the lower plate and the periodicity conditions (12) yield

$$
\begin{gathered}
\partial_{z} \phi=0 \text { at } z=-d, \\
\phi\left(x+l_{1}, y, z, l\right)=\phi\left(x, y+l_{2}, z, l\right)=\phi(x, y, z, t) \\
h^{\circ}\left(x+L_{1}, y, t\right)=h^{\circ}\left(x, y+L_{2}, t\right)=h^{o}(x, y, t) .
\end{gathered}
$$

We are consistently neglecting terms of order $\varepsilon^{3}$ in Eqs. (31) and (32) because of the approximations listed in Eq. (15), and taking into account that those terms that are cither (a) cubic in the oscillatory flow variables or (b) lincar in both an oscillatory variable and a slowly varying variable, cxhibit zcro temporal mean values at leading order.

Before procecding any further, we note that mean llow does not contribute to the averaged (in the time scale $t \sim 1$ ) energy equation at leading order, which is consistent with the fact that mean flow variables (velocity and free surface deflection) are small compared to their counterparts in the oscillatory flow. The averaged energy equation is obtained upon multiplication of Eq. (26) by $\partial_{t} \phi$, integration in $0<x<L_{1}, 0<y<L_{2},-d<z<0$, averaging over a period of oscillation, integration by parts repeatedly, and substitution of Eqs. (31), (32), and (34). We find

$$
\begin{aligned}
\frac{d \mathcal{E}}{d t}= & -\int_{0}^{L_{1}} \int_{0}^{L_{2}} \gamma\left\langle\partial_{z}\left(|\boldsymbol{\nabla} \phi|^{2}+\left|\partial_{z} \phi\right|^{2}\right)+8 a\left(\partial_{l^{\prime}}\right)\right. \\
& \left.\times\left\langle h^{\circ} \sin 2 l\right\rangle^{t o}\right\rangle^{t s} d x d y+O\left(\gamma+a+\varepsilon^{2}\right)
\end{aligned}
$$

where $\varepsilon$ is the time-averaged (kinetic plus potential) energy. The first term on the right-hand side of Eq. (35) (except for a factor of 2) is the classical result, first given by Landau and Lifshitz [29]. that approximated viscous dissipation by linear damping from the bulk potential flow (see also $[10,11]$ ). Note that mean flow variables (both velocity and free surface deflection) are small compared to their counterparts in the oscillatory flow and do not contribute to the energy at leading order. To obtain Eq. (35), we have taken into account that $\boldsymbol{u}^{m}$ and $w^{m}$ are independent of $t$ at leading order, and that if $\psi$ and $\varphi$ are $t$-periodic, of period $2 \pi$ (as the variables associatcd with the oscillatory flow are to first approximation), then to leading order we have $\left.\left\langle\varphi \int^{t} \psi\right\rangle^{s s}=-\left\langle\psi \int^{t} \psi\right\rangle\right\rangle^{t s}$ and $\left.\left\langle\psi \int^{t} \psi\right\rangle\right\rangle^{t s}=0$.

\section{Mean flow}

In order to obtain the equations and boundary conditions governing the slowly varying flow, we must take into account the oscillatory boundary layer attached to the free surface, which provides (at the edge of this layer) a slowly varying shear stress that must be imposed as a boundary condition for the mean flow in the bulk. This forcing mechanism was first uncovered by Longuet-Higgins [23], who obtained an explicit expression for the forcing shear stress produced by general boundary layers in 2D. The counterpart of this expression in 3D (for a frec surface of general shape) has only been obtained quite recently [30], although quasi-planar frec surfaces (such as the ones considered here) were already considered in a not 100 well known work [31]. With the notation of this paper, the general formulas derived in [30] yield

$$
\begin{aligned}
\partial_{z} \boldsymbol{u}^{m}+\nabla w^{m}= & 2\left\langle\boldsymbol{\nabla}\left[\boldsymbol{\nabla} \cdot\left(h^{o} \boldsymbol{\nabla} \phi\right)\right]+\left(\boldsymbol{\nabla} h^{o} \cdot \boldsymbol{\nabla}\right) \boldsymbol{\nabla} \phi\right. \\
& \left.+\left(\boldsymbol{\nabla}^{2} \phi\right) \boldsymbol{\nabla} h^{o}\right\rangle^{t s} \text { al } z=0,
\end{aligned}
$$

where only the leading-order contribution as $\gamma \rightarrow 0$ and $\varepsilon$ $\rightarrow 0$ is retained. The boundary layer attached to the free sur- 
face has no effect on the other two boundary conditions at the unperturbed free surface, which are directly obtained from Eqs. (8) and (13) to be

$$
\begin{gathered}
p^{m}-\left(1-\Gamma^{\top}\right) h^{m}+\mathbf{I} \nabla^{2} h^{m}=\left\langle h^{o} \partial_{t z}^{2} \phi+\left(|\boldsymbol{\nabla} \phi|^{2}+\left|\partial_{z} \phi\right|^{2}\right) / 2\right\rangle^{\langle s}, \\
\partial_{l} h^{m}+\boldsymbol{\nabla} \cdot\left(\int_{-d}^{0} \boldsymbol{u}^{m} d z\right)=-\boldsymbol{\nabla} \cdot\left(\left\langle h^{o} \nabla \phi\right\rangle^{t s}\right) \text { at } z=0,
\end{gathered}
$$

where we are only taking into account the lcading-order terms. And from Eq. (5) we have no slip at the lower plate,

$$
\boldsymbol{u}^{m}=0, \quad w^{m}=0 \text { al } z=-d,
$$

at leading order. We are neglecting the effect of the oscillatory boundary layer attached to the lower plate because its effect is quite small (the horizontal component of the mean flow velocity near the lower plate is proportional to the square of the vertical jump of the horizontal component of the oscillatory velocity accross the lower boundary layer [28], which is $O\left(\mathrm{c}^{2 d}\right) \varepsilon^{2} \ll \varepsilon^{2}$ [Eq. (14)]; this in turn is small compared to the streaming flow velocity in the bulk, which is $O\left(\varepsilon^{2}\right)$ ). These boundary conditions show that mean flow is forced by surface waves in two ways. Those terms appearing on the right-hand sides of Eqs. (37) and (38) provide an inviscid forcing mechanism that by itself would provide an inviscid mean flow, like that appearing in the DaveyStewartson model [22]. The right-hand side of Eq. (36) instcad produces a forcing shear stress that drives a viscous mean flow, which is absent in the usual inviscid and ncarly inviscid theorics of Faraday waves. Note that his forcing stress is generically nomzero and independent of viscosity at leading order, a fact that is well known but somewhat surprising because this effect is due to the oscillatory boundary layer, and is absent in the strictly inviscid case. We remind the reader that the limit of vanishing viscosity is a singular limit which does commute with the limit $\varepsilon \rightarrow 0$. We could decompose the mean flow into its inviscid and viscous parts, as is done in $[13,14]$, but for convenience this is not done here.

Finally, we substitute Eqs. (18) and (25) into Eqs. (2) and (3), and take the time average defined in Eq. (20) in the resulting equations. Proceeding as we did to obtain Eq. (35), we find, after some algebra,

$$
\begin{gathered}
\boldsymbol{\nabla} \cdot \boldsymbol{u}^{m}+\partial_{z} w^{m}=0 \\
\partial_{t} \boldsymbol{u}^{m}-\varepsilon^{2}\left[\left(w^{S d}+w^{m}\right)\left(\boldsymbol{\nabla} w^{m}-\partial_{z} \boldsymbol{u}^{m}\right)+\left(\boldsymbol{u}^{S d}+\boldsymbol{u}^{m}\right)-\boldsymbol{\nabla} \cdot \boldsymbol{u}^{m \perp}\right] \\
=-\boldsymbol{\nabla} q^{m}+\gamma\left(\boldsymbol{\nabla}^{2} \boldsymbol{u}^{m}+\partial_{z z}^{2} \boldsymbol{u}^{m}\right) / 2 \\
\partial_{t} w^{m}+\varepsilon^{2}\left(\boldsymbol{u}^{S d}+\boldsymbol{u}^{m}\right) \cdot\left(\nabla w^{m}-\partial_{z} \boldsymbol{u}^{m}\right) \\
=-\partial_{z} q^{m}+\gamma\left(\nabla^{2} w^{m}+\partial_{z z}^{2} w^{m}\right) / 2
\end{gathered}
$$

where $\boldsymbol{u}^{S d}$ and $w^{S d}$ are the horizontal and vertical components of the Stokes drift given by Eq. (22), and the modified pressurc $q^{n}$ is defined as

$$
q^{n}=p^{m}+\varepsilon^{2}\left\langle\partial_{z} \phi\left(\int^{t} \nabla \phi\right) \cdot\left(\nabla w^{m}-\partial_{z} u^{m}\right)\right\rangle^{t s} .
$$

Finally the periodicity condition (12) yields

$$
\begin{aligned}
& \left(\boldsymbol{u}^{m}, w^{m}, q^{m}\right)\left(x+L_{1}, y, z, t\right)=\left(\boldsymbol{u}^{m}, w^{m}, q^{m}\right)\left(x, y+L_{2}, z, t\right) \\
& =\left(\boldsymbol{u}^{m}, w^{m}, q^{m}\right)(x, y, z, t) \text {, } \\
& h^{m}\left(x+L_{1}, y, t\right)=h^{m}\left(x, y+L_{2}, t\right)=h^{m}(x, y, t) .
\end{aligned}
$$

In order to estimate the magnitude of the various terms that depend on the surface-wave variables and force the mean flow, we assume that at leading order the velocity potential $\phi$ and the free-surface deflection $h^{\circ}$ can be written as a superposition of plane waves,

$$
\begin{gathered}
\phi=i e^{z} \sum_{n=-N}^{N} A_{n} e^{i\left(l \cdot \mathbf{k}_{n} \cdot \mathbf{x}\right)}+\mathrm{c} . \mathrm{c} .+\cdots, \\
h^{o}=\sum_{n=-N}^{N} A_{n} e^{i\left(t+\mathbf{k}_{n} \cdot \mathbf{x}\right)}+\text { c.c. }+\cdots
\end{gathered}
$$

where the complex amplitudes $A_{-N}, \ldots, A_{N}$ are allowed to depend only on slow space and time variables. The wave vectors $\mathbf{k}_{-N}, \ldots, \mathbf{k}_{N}$ correspond to only $N$ directions because they are related in pairs as

$$
\mathbf{k}_{-n}=-\mathbf{k}_{n} \quad \text { and } \quad\left|\mathbf{k}_{n}\right|=1 \text { for } n=1, \ldots, N .
$$

Thus for each $n=1, \ldots, N$, the $n$th and the $(-n)$ th waves counterpropagate along the same direction. Note that each pair of counterpropagating waves builds a standing wave in the short time scale if and only if $\left|A_{n}\right|=\left|A_{n}\right|$, and the whole surface-wave pattern is seen to be standing in the short time scale if and only if the following, more stringent condition holds:

$$
A_{m t} A_{n}=A_{-n t} A_{-n} \text { for all } m, n=1, \ldots, N .
$$

Such surface waves will be called quasistanding below.

By using Eq. (45), the forcing terms in the boundary conditions Eqs. (36)-(38) are wrilten as

$$
\begin{aligned}
& \left\langle 2 h^{\prime \prime} \partial_{t z}^{2} \phi+|\nabla \phi|^{2}+\left|\partial_{\bar{z}} \phi\right|^{2}\right\rangle^{k s} \\
& =\left\langle|\nabla \phi|^{2}-\left|\partial_{z} \phi\right|^{2}\right\rangle^{t s} \\
& =\sum_{m, n}\left(\mathbf{k}_{m} \cdot \mathbf{k}_{n}-1\right) A_{m} A_{n} e^{i\left(\mathbf{k}_{n} \mathbf{k}_{m}\right) \cdot \mathbf{x}} \\
& +\sum_{m, n} i\left(A_{n} \mathbf{k}_{n} \cdot \boldsymbol{\nabla} \overline{A_{m}}-\overline{A_{m}} \mathbf{k}_{m} \cdot \boldsymbol{\nabla} A_{n}\right) e^{i\left(\mathbf{k}_{n} \mathbf{k}_{m}\right) \cdot \mathbf{x}}+\text { c.c. } \\
& +O\left(L^{2}\right) \\
& \nabla \cdot\left\langle h^{\prime \prime} \nabla \phi\right\rangle^{k s}=\left\langle\boldsymbol{\nabla} \cdot h^{\prime} \nabla \phi\right\rangle^{l s} \\
& =-2 \sum_{m} \mathbf{k}_{m} \cdot \nabla\left(\left|A_{m}\right|^{2}\right)+O\left(L^{-2}\right),
\end{aligned}
$$




$$
\begin{aligned}
& \left\langle\boldsymbol{\nabla}\left[\boldsymbol{\nabla} \cdot\left(h^{\circ} \boldsymbol{\nabla} \phi\right)\right]+\left(\boldsymbol{\nabla} h^{o} \cdot \boldsymbol{\nabla}\right) \boldsymbol{\nabla} \phi+\left(\boldsymbol{\nabla}^{2} \phi\right) \nabla h^{o}\right\rangle^{t s} \\
& =\sum_{m_{n} n}\left(\mathbf{1}+\mathbf{k}_{m} \cdot \mathbf{k}_{n}\right) \mathbf{k}_{m} A_{m} A_{n} e^{i\left(\mathbf{k}_{n} \mathbf{k}_{m}\right) \cdot \mathbf{x}} \\
& \quad+\text { c.c. }+O\left(L^{-1}\right),
\end{aligned}
$$

at $z=0$; and the Stokes drift velocily components in Eq. (22) are writlen as

$\boldsymbol{u}^{i d t}=\omega^{1} e^{2 z} \sum_{m, n}\left(\mathbf{k}_{m} \cdot \mathbf{k}_{n}+1\right) \mathbf{k}_{m} \bar{A}_{m} A_{n} e^{i\left(\mathbf{k}_{n} \mathbf{k}_{m}\right) \cdot \mathbf{x}}+$ c.c. $+O\left(L^{1}\right)$

$$
w^{S i d}=2 \omega{ }^{1} e^{2 \Sigma} \sum_{m} \mathbf{k}_{m} \cdot \nabla\left(\left|A_{m}\right|^{2}\right)+O\left(L^{2}\right)
$$

in $-d<z<0$.

Several remarks can be made about these boundary conditions. First, the forcing term given in Eq. (48) depends only weakly on the horizontal variables $x$ and $y$, namely

$$
\left\langle\left\langle 2 h^{o} \partial_{t z}^{2} \phi+|\nabla \phi|^{2}+\left|\partial_{z} \phi\right|^{2}\right\rangle^{t s}\right\rangle^{h s} \sim L^{-1}
$$

where $\langle\cdot\rangle^{h s}$ is the horizontal average in the short spatial scales

$$
\langle\psi \mid\rangle^{h s}=\left(\int_{B} d x d y\right)^{-1} \int_{B} \psi d x d y
$$

Here $\mathcal{B}$ is a ball of radius large compared to 1 but small compared to $L$. Or, in terms of a horizontal Fourier transform with associated wave numbers $\mathbf{k}_{m n} \neq 0$ if $(m, n) \neq(0,0)$, with $\mathbf{k}_{\text {(10) }}=\mathbf{0}$,

$$
\langle\psi\rangle^{h s}=\psi_{00} \text { if } \psi=\sum_{m_{3}, n} \psi_{m n} e^{i \mathbf{k}_{m n} \mathbf{x}}
$$

where $\psi_{m n}$ is allowed to depend weakly on $x, y$, and $t$ (and strongly on $z$ ).

Second, the forcing terms in Eqs. (49) and (50) and the Stokes drift vanish at leading order if the surface-wave pattern is quasistanding.

$$
\begin{aligned}
&\left\langle\boldsymbol{\nabla}\left[\boldsymbol{\nabla} \cdot\left(h^{\prime} \boldsymbol{\nabla} \phi\right)\right]+\left(h^{\prime} \boldsymbol{\nabla} \cdot \boldsymbol{\nabla}\right) \boldsymbol{\nabla} \phi+\left(\nabla^{2} \phi\right) \nabla h^{\prime}\right)^{k} \\
& \sim \boldsymbol{u}^{S d} \sim L^{-1},
\end{aligned}
$$$$
\text { and } \nabla \cdot\left\langle h^{o} \nabla \phi\right\rangle^{t s} \sim w^{S t} \sim L^{-2} \text { if Eq. (47) holds. }
$$

Third, and according to Eqs. (49) and (52), we have

$$
\left|\left[\left\langle\boldsymbol{\nabla} \cdot\left(h^{o} \boldsymbol{\nabla} \phi\right)\right\rangle^{s}\right]_{z=0}\right|=O\left(L^{1}\right), \quad\left|w^{s i d}\right|=O\left(L^{1}\right)
$$

By using the continuity equation (40), the boundary condition (38) can be rewritten as $\partial_{t} h^{m}+w^{m}+\left\langle h^{o} \nabla \phi\right\rangle^{t s}=0$, which invoking Eqs. (21) and (57) yiclds, at leading order,

$$
w^{n} \sim L^{-1} \text { at } z=0 \text {. }
$$

\section{Short- and long-wave decomposition of the mean flow}

We next decompose the mean flow variables into a shortwowe component (or oscillatory in the horizontal direction) and a long-wave component (or slowly varying in the horizontal direction)

$$
\begin{aligned}
\left(\boldsymbol{u}^{m}, w^{m}, q^{m}, h^{m}\right)= & \left(\boldsymbol{u}^{m o}, w^{m o}, \varepsilon^{2} q^{m o}, h^{m o}\right) \\
& +\left(\boldsymbol{u}^{m s}, \varepsilon^{2} w^{m s}, q^{m s}, h^{m s}\right),
\end{aligned}
$$

where the short-wave component is such that

$$
\left\langle\boldsymbol{u}^{m o}\right\rangle^{h s}=0, \quad\left\langle w^{m o}\right\rangle^{h s}=\left\langle q^{m o}\right\rangle^{h s}=\left\langle h^{m o}\right\rangle^{h s}=0 .
$$

The long-wave components depend weakly on the horizontal variables

$$
\begin{aligned}
\left|\boldsymbol{\nabla} \boldsymbol{u}^{m s}\right| \sim \varepsilon^{2}\left|\boldsymbol{u}^{m s}\right|, & \left|\boldsymbol{\nabla} w^{m s}\right| \sim \varepsilon^{2}\left|w^{m s}\right|, & \left|\boldsymbol{\nabla} p^{m s}\right| \sim \varepsilon^{2}\left|p^{m s}\right|, \\
& \left|\boldsymbol{\nabla} h^{m s}\right| \sim \varepsilon^{2}\left|h^{m s}\right|, &
\end{aligned}
$$

where we are assuming that the slow spatial scale for horizontal gradients of the long-wave mean flow is the same as that of the envelope of surface waves, namely of the order of $l, \sim \varepsilon^{2}$ [sce Eq. (15)]. Also, in Eq. (59) $\boldsymbol{u}^{m o} \sim w^{m o} \sim q^{m o}$ $\sim h^{m w s} \sim \boldsymbol{u}^{m s} \sim w^{m s} \sim q^{m s} \sim h^{m s} \sim 1$ and thus we are anticipaling the order of magnitude of all variables associated with both mean flow components.

The equations governing the short-wave component of the mean flow are obtained by substituting Eq. (59) into Eqs. (36)-(42). The short-wave deflection $h^{\text {no }}$ is given by

$$
\begin{aligned}
-(1-\Gamma) h^{m o}+\Gamma \nabla^{2} h^{m o}= & {\left[\left\langle\left\langleh^{\circ} \partial_{t z}^{2} \phi+\left(|\nabla \phi|^{2}\right.\right.\right.\right.} \\
& \left.\left.\left.\left.+\left|\partial_{z} \phi\right|^{2}\right) / 2\right\rangle^{t s}\right\rangle^{h o}\right]_{z-0},
\end{aligned}
$$

with periodic boundary conditions $h^{\prime \prime}\left(x+l_{1}, y, l\right)=h^{\prime \prime}(x, y$ $\left.+l_{2}, l\right)=h^{\circ}(x, y, l)$, and where \langle\rangle$^{h o}$ denotes the short-wave component

$$
\langle\psi\rangle^{h u}=\psi-\langle\psi\rangle^{h s}
$$

Note that, according to Eq. (53), the horizontal mean value of the right-hand side of Eq. (62) vanishes at leading order, as required by volume conservation.

Short-wave velocity and pressure $\boldsymbol{u}^{m o}, w^{m o}$, and $q^{m o}$ are given by

$$
\boldsymbol{\nabla} \cdot \boldsymbol{u}^{m o}+\partial_{z} w^{m o}=0
$$

$$
\begin{aligned}
\partial_{t} \boldsymbol{u}^{m o}- & \varepsilon^{2}\left\langle w^{m o}\left(\nabla w^{m o}-\partial_{z} \boldsymbol{u}^{m o}-\partial_{z} \boldsymbol{u}^{m s}\right)\right. \\
& \left.+\left(\boldsymbol{u}^{S d}+\boldsymbol{u}^{m o}+\boldsymbol{u}^{m s}\right)-\boldsymbol{\nabla} \cdot \boldsymbol{u}^{m o}\right\rangle^{h o} \\
= & -\varepsilon^{2} \boldsymbol{\nabla} q^{m o}+\gamma\left(\nabla^{2} \boldsymbol{u}^{m o}+\partial_{z z}^{2} \boldsymbol{u}^{m o}\right) / 2
\end{aligned}
$$

$$
\begin{gathered}
\partial_{t} w^{m o}+\varepsilon^{2}\left\langle\left(\boldsymbol{u}^{S d}+\boldsymbol{u}^{m o}+\boldsymbol{u}^{m s}\right) \cdot\left(\boldsymbol{\nabla} w^{m o}-\partial_{z} \boldsymbol{u}^{m o}-\partial_{z} \boldsymbol{u}^{m s}\right)\right\rangle^{h o} \\
=-\varepsilon^{2} \partial_{z} q^{m o}+\gamma\left(\nabla^{2} w^{m o}+\partial_{z z}^{2} w^{m o}\right) / 2
\end{gathered}
$$

in $-d<z<0$, with boundary conditions

$$
\begin{gathered}
\boldsymbol{u}^{m o}=0, \quad w^{m o}=0 \text { at } z=-d \\
\partial_{z} \boldsymbol{u}^{m o}=2\left\langle\left\langle\left(\nabla h^{\circ} \cdot \nabla\right) \nabla \phi+\left(\nabla^{2} \phi\right) \nabla h^{o}\right\rangle^{t s}\right\rangle^{h o}, \\
w^{m o}=0 \text { at } z=0,
\end{gathered}
$$

$$
\begin{aligned}
\left(\boldsymbol{u}^{m o}, w^{m 0}, q^{m o}\right)\left(x+L_{1}, y, z, t\right) & =\left(\boldsymbol{u}^{m o}, w^{m o t}, q^{m o}\right)\left(x, y+L_{2}, z, t\right) \\
& =\left(\boldsymbol{u}^{m o}, w^{m o t}, q^{m o}\right)(x, y, z, t),
\end{aligned}
$$




$$
h^{m o}\left(x+L_{1}, y, t\right)=h^{m o}\left(x, y+L_{2}, t\right)=h^{m o}(x, y, t),
$$

where we have taken into account Eq. (57). Note that this problem is decoupled from that giving $h^{m o}$ [Eq. (62)].

In order to determine the equations governing the longwave component of the mean flow, we first take into account that, according to the continuity equation and the boundary condition (39), the rescaled vertical velocity component is given by

$$
w^{m s}=-\varepsilon^{-2} \int_{-d}^{z} \nabla \cdot u^{m s} d z
$$

and according to Eq. (61), is of order unity as assumed above. The problem giving $\left(\boldsymbol{u}^{m s}, q^{m s}, h^{m s}\right)$ becomes decoupled from $w^{m s}$ as we show now. From the momentum equation (42) we obtain

$$
q^{m s}=q^{m s}(x, y, t),
$$

and then, by invoking Eq. (57), the momentum equation (41) yiclds

$$
\begin{gathered}
\partial_{l} \boldsymbol{u}^{m s}-\varepsilon^{2}\left\langle w^{m o}\left(\nabla w^{m o}-\partial_{z} \boldsymbol{u}^{m o}\right)+\left(\boldsymbol{u}^{S d}+\boldsymbol{u}^{m o}\right)^{\perp} \nabla \cdot \boldsymbol{u}^{m o}\right)^{h s} \\
=-\boldsymbol{\nabla} q^{m s}+\gamma \partial_{z z} \boldsymbol{u}^{m s} / 2
\end{gathered}
$$

in $-d<z<0$, with boundary conditions

$$
\begin{gathered}
\boldsymbol{u}^{m s}=0 \text { at } z=-d, \\
\partial_{z} \boldsymbol{u}^{m s s}=2\left\langle\left\langle\left(\boldsymbol{\nabla} h^{o} \cdot \boldsymbol{\nabla}\right) \boldsymbol{\nabla} \phi+\left(\boldsymbol{\nabla}^{2} \phi\right) \boldsymbol{\nabla} h^{o}\right\rangle^{t s}\right\rangle^{h s}, \\
q^{m i s}-(1-\Gamma) h^{m s}=0, \\
\partial_{t} h^{m s}+\boldsymbol{\nabla} \cdot\left(\int_{-d}^{0} \boldsymbol{u}^{m s} d z\right)=-\nabla \cdot\left(\left\langle\left\langle h^{o} \nabla \phi\right\rangle^{t s}\right\rangle^{h s}\right),
\end{gathered}
$$

at $z=0$, resulting from Eqs. (36)-(39) and (43). The periodic boundary conditions (12) lead to

$$
\begin{aligned}
\left(\boldsymbol{u}^{m n s}, w^{m s s}, q^{m s}\right)\left(x+L_{1}, y, z, t\right) & =\left(\boldsymbol{u}^{m s}, w^{m s s}, q^{m s}\right)\left(x, y+L_{2}, z, t\right) \\
& =\left(\boldsymbol{u}^{m s}, w^{m s s}, q^{m s}\right)(x, y, z, t), \\
h^{m n s}\left(x+L_{1}, y, t\right) & =h^{m s s}\left(x, y+L_{2}, t\right) \\
& =h^{m s}(x, y, t) .
\end{aligned}
$$

All terms appearing in Eqs. (72)-(74) are of the same order because $\gamma \sim \varepsilon^{2}$ [see Eq. (15)] and $\boldsymbol{u}^{m s}, q^{m s s}$, and $h^{m s}$ satisfy Eqs. (21) and (61). The forcing term in Eq. (74)b $\boldsymbol{\nabla} \cdot\left\langle\left\langle h^{\prime} \nabla \phi\right\rangle^{\prime s}\right\rangle^{h s}$ is inviscid, and is the only forcing term that appears in the standard Davey-Stewartson model [22] which involves a potential mean flow. This model is only valid for stripes patterns as described below in Eqs. (83)-(86), where this term is precisely the only forcing term remaining on the right-hand side of Eq. (86). On the other hand, the forcing terms on the right-hand sides of Eqs. (68) and (73) are necessarily viscous and drive a vortical flow.

Substituting Eqs. (59) into Eqs. (26)-(33), we obtain the following equation (after some algebra):

$$
\begin{aligned}
\nabla^{2} \phi & +\partial_{z z}^{2} \phi+\varepsilon^{2}\left(\int^{t} \partial_{z} \phi\right) \nabla \cdot\left(\nabla w^{m o}-\partial_{z} \boldsymbol{u}^{m o}\right) \\
& +\varepsilon^{2} \nabla \cdot\left[\left(\int^{t} \boldsymbol{\nabla} \phi\right)^{\perp} \boldsymbol{\nabla} \cdot \boldsymbol{u}^{m o \perp}\right] \\
& -\varepsilon^{2}\left(\int^{t} \boldsymbol{\nabla} \phi\right) \cdot \partial_{z}\left(\nabla w^{m o}-\partial_{z} \boldsymbol{u}^{m o}-\partial_{z} \boldsymbol{u}^{m 5}\right)=0
\end{aligned}
$$

for $-d<z<0$, and boundary conditions

$$
\begin{aligned}
\partial_{t} h^{o} & -\partial_{z} \phi+\varepsilon^{2}\left(\int^{t} \nabla \phi\right) \cdot\left(\boldsymbol{\nabla} w^{m o}-\partial_{z} \boldsymbol{u}^{m o}-\partial_{z} \boldsymbol{u}^{m s}\right) \\
& +\varepsilon\left\langle\boldsymbol{\nabla} \cdot\left(h^{o} \nabla \phi\right)\right\rangle^{t o}+\varepsilon^{2} \boldsymbol{\nabla} \cdot\left[\left(h^{m o}+h^{m s}\right) \boldsymbol{\nabla} \phi+h^{o}\left(\boldsymbol{u}^{m o}\right.\right. \\
& \left.\left.+\boldsymbol{u}^{m s}\right)+\left(h^{o}\right)^{2} \partial_{z} \boldsymbol{\nabla} \phi / 2\right]=\gamma \int^{t} \boldsymbol{\nabla}^{2}\left(\partial_{z} \phi\right)
\end{aligned}
$$

$$
\begin{aligned}
& \partial_{t} \phi+\varepsilon\left\langle h^{\prime \prime} \partial_{t z}^{2} \phi\right\rangle^{\prime \prime \prime}+\varepsilon^{2}\left[\left(h^{m \omega \prime}+h^{m s}\right) \partial_{t z}^{2} \phi-h^{\prime \prime} \partial_{z} \mathcal{A}^{m s}\right. \\
& \left.+\left(h^{\circ}\right)^{2} \partial_{t z z}^{3} \phi / 2\right]+\varepsilon\left\langle|\nabla \phi|^{2}+\left|\partial_{z} \phi\right|^{2}\right\rangle^{t o} / 2+\varepsilon^{2}\left[\left(\boldsymbol{u}^{m o}\right.\right. \\
& \left.\left.+\boldsymbol{u}^{m i s}\right) \cdot \boldsymbol{\nabla} \phi+h^{\prime \prime} \partial_{z}\left(|\boldsymbol{\nabla} \phi|^{2}+\left|\partial_{\bar{z}} \phi\right|^{2}\right) / 2\right]+4 a\left\langle h^{o} \sin 2 t\right\rangle^{t o} \\
& +(1-\Gamma) h^{o}-\Gamma \boldsymbol{\nabla} \cdot\left[\boldsymbol{\nabla} h^{\prime \prime} /\left(1+\varepsilon^{2}\left|\nabla h^{\prime \prime}\right|^{2}\right)^{1 / 2}\right]+\gamma \partial_{z z}^{2} \phi=0,
\end{aligned}
$$

$$
\partial_{z} \phi=0 \text { at } z=-d .
$$

This is the central result of this section. In the limit considered [Eq. (15)], llow variables have been decomposed into oscillatory and slowly varying parts [Eq. (18)], where the oscillatory components are given by Eq. (25), with $\phi$ and $h^{\circ}$ given by Eqs. (26)-(34). The slowly varying component has been further decomposed into short-wave and long-wave components in Eq. (59), with the short-wave component given by $\mathrm{Eq}$. (62), and the long-wave one given by Eqs. (72)-(74). Thus the coupled evolution of surface waves and mean flow is given by Eqs. (62), (64)-(69), and (72)-(79). This system of equations still includes the full 3D NavierStokes equations (65) and (66) with a large Reynolds number based on the horizontal size [although an $O(1)$ Reynolds number is based on the container depth].

The numerical solution of this coupled problem remains quite complicated, and further simplifications are necessary to make it tractable. We discuss in the following section a hierarchy of simplified models that are based on various physical assumptions and in some cases on ad hoc approximations.

\section{APPROXIMATIONS TO THE COUPLED MEAN-FLOW-SURFACE-WAVE EQUATIONS}

\section{A. Stripes}

In this particular case, the equations derived above simplify substantially. Unfortunately in the nearly inviscid limit we are considering stripes are not the selected pattern in 3D [32], except in the purely gravity wave limit of $I \ll 1$. This 
would require that the basic wavelength $2 \pi / k^{*}$ be large compared to the capillary length $\ell_{c}=\sqrt{ } \sigma /\left(\rho g_{0}\right)$. which is of the order of $3 \mathrm{~mm}$ for water. We consider first the strict gravitational limit of $\Gamma=0$. If only one stripe is present at each point, then we can take $N=1$ in Eqs. (49)-(52) at this point, and the short-wave parts of $\boldsymbol{u}^{\text {sit }}$ and $\left\langle\left(\nabla h^{\prime \prime} \cdot \nabla\right) \nabla \phi\right.$ $\left.+\left(\nabla^{2} \phi\right) \nabla h^{o}\right\rangle^{t s}$ vanish in Eqs. (64)-(68). This implics that the short-wave part of the mean flow is unforced, and thus admits the solution $\boldsymbol{u}^{m o}=0, w^{m o}=0, q^{m o}=$ const which we assume is globally stable. Then for large times such that the short-wave component vanishes, the remaining variables are given by Eqs. (26), (31)-(33), (62), and (72)-(74). By also invoking Eq. (61), these latter equations can be rewritten as

$$
\begin{gathered}
\nabla^{2} \phi+\partial_{z z}^{2} \phi+\varepsilon^{2}\left(\int \boldsymbol{\nabla} \phi\right) \cdot \partial_{z \Sigma} \boldsymbol{u}^{m s s}=0 . \\
\partial_{t} \boldsymbol{u}^{m s s}=-\nabla h^{m s s}+\gamma \partial_{z \Sigma} \boldsymbol{u}^{m s / 2}
\end{gathered}
$$

in $-d<z<0$, with boundary conditions

$$
\partial_{z} \phi=\boldsymbol{u}^{m s}=0
$$

at $z=-d$ and

$$
\begin{aligned}
& \partial_{t} h^{o}-\partial_{z} \phi-c^{2}\left(\int \nabla_{\phi}\right) \cdot \partial_{z} \boldsymbol{u}^{m s}+c\left\langle\boldsymbol{\nabla} \cdot\left(h^{o} \boldsymbol{\nabla} \phi\right)\right\rangle^{t o} \\
& +r^{2} \boldsymbol{\nabla} \cdot\left[\left(h^{m o}+h^{m s}\right) \boldsymbol{\nabla} \phi+h^{o} u^{m s}+\left(h^{o}\right)^{2} \partial_{\underline{z}} \boldsymbol{\nabla} \phi / 2\right] \\
& =\gamma \int^{t} \nabla^{2}\left(\partial_{z} \phi\right) \\
& \partial_{t} \phi+\varepsilon\left\langle h^{o} \partial_{i z}^{2} \phi\right\rangle^{l o}+\varepsilon^{2}\left[\left(h^{m o}+h^{m s}\right) \partial_{t z}^{2} \phi-\partial_{z} q^{m s}+\left(h^{\prime}\right)^{2} \partial_{l z z}^{3} \phi / 2\right] \\
& +\varepsilon\left\langle|\nabla \phi|^{2}+\left|\partial_{z} \phi\right|^{2}\right\rangle^{\omega \prime} / 2+\varepsilon^{2}\left[u^{m x} \cdot \nabla \phi+h^{o} \partial_{z}\left(|\nabla \phi|^{2}\right.\right. \\
& \left.\left.+\left|\partial_{z} \phi\right|^{2}\right) / 2\right]+4 a\left\langle h^{\prime \prime} \sin 2 t\right\rangle^{\prime \prime \prime}+h^{\prime \prime}+\gamma \dot{\partial}_{z z}^{2} \phi=0, \\
& \partial_{\bar{z}} \boldsymbol{u}^{m s}=2\left\langle\left\langle\left(\boldsymbol{\nabla} h^{\prime \prime} \cdot \boldsymbol{\nabla}\right) \boldsymbol{\nabla} \phi+\left(\boldsymbol{\nabla}^{2} \phi\right) \nabla h^{\prime}\right\rangle^{\Delta s}\right\rangle^{h s}, \\
& \partial_{t} h^{m s}+\nabla \cdot\left(\int_{-d}^{0} \boldsymbol{u}^{m s} d z\right)=-\nabla \cdot\left(\left\langle\left\langle h^{o} \nabla \phi\right\rangle^{t s}\right\rangle^{h s}\right),
\end{aligned}
$$

at $z=0$. The periodic boundary conditions in the horizontal variables resulting from $\mathrm{Eq}$. (12) are

$$
\begin{aligned}
\left(\phi, h^{o}\right)\left(x+L_{1,}, y, z, t\right) & =\left(\phi, h^{o}\right)\left(x, y+L_{2}, z, t\right) \\
& =\left(\phi, h^{o}\right)(x, y, z, t), \\
\left(h^{o}, h^{m o}, h^{m s}\right)\left(x+l_{11}, y, z, l\right) & =\left(h^{o}, h^{m o}, h^{m s}\right)\left(x, y+l_{2}, z, t\right) \\
& =\left(h^{o}, h^{m o}, h^{m s}\right)(x, y, z, t) .
\end{aligned}
$$

These equations are considerably simpler as they only include the heat equation, Eq. (81), instead of the full continuity and Navier-Stokes equations, but yet allow significant variation in stripe orientation.

\section{B. Linear approximation for the mean flow}

The mean flow cquations and boundary conditions Eqs. (64)-(68) and (72)-(74) are linear in the mean flow variables except for convecive terms. If these are neglected, then the mean flow equations are rewritten as Eq. (62) and

$$
\begin{aligned}
& \boldsymbol{\nabla}^{2} \phi+\partial_{s z}^{2} \phi+\varepsilon^{2}\left(\int \partial_{\varepsilon} \phi\right) \nabla \cdot\left(\nabla w^{m(x)}-\partial_{\varepsilon} \boldsymbol{u}^{m(j)}\right. \\
& +\varepsilon^{2} \boldsymbol{\nabla}\left[\left(\int^{t} \nabla \phi\right)^{\prime} \boldsymbol{\nabla} \cdot u^{m o \perp}\right]-\varepsilon^{2}\left(\int^{t} \boldsymbol{\nabla} \phi\right) \cdot \partial_{z}\left(\boldsymbol{\nabla} w^{m o}\right. \\
& \left.-\partial_{z} u^{m o}-\partial_{z} u^{m s}\right)=0 \text {, } \\
& \boldsymbol{\nabla} \cdot \boldsymbol{u}^{m o}+\partial_{z} w^{m o \prime}=0 \\
& \partial_{l} \boldsymbol{u}^{m o}=-\varepsilon^{2} \nabla q^{m o}+\gamma\left(\nabla^{2} \boldsymbol{u}^{m o}+\partial_{z=}^{2} \boldsymbol{u}^{m o}\right) / 2, \\
& \partial_{1} w^{m o}=-\varepsilon^{2} \partial_{z} q^{m o}+\gamma\left(\nabla^{2} w^{m o}+\partial_{z \Sigma}^{2} w^{m o}\right) / 2, \\
& \partial_{l} \boldsymbol{u}^{m s}=-\boldsymbol{\nabla} q^{m s}+\gamma \partial_{z z} \boldsymbol{u}^{m s} / 2,
\end{aligned}
$$

in $-d<z<0$, with boundary conditions

$$
\partial_{z} \phi=0, \quad \boldsymbol{u}^{m o}=\boldsymbol{u}^{m s s}=0, \quad w^{m o}=w^{m s}=0,
$$

at $z=-d$, and

$$
\begin{aligned}
\partial_{i} h^{o} & -\partial_{z} \phi+\varepsilon^{2}\left(\int^{t} \boldsymbol{\nabla} \phi\right) \cdot\left(\boldsymbol{\nabla} w^{m o}-\partial_{z} \boldsymbol{u}^{m o}-\partial_{z} \boldsymbol{u}^{m s}\right) \\
& +\varepsilon\left\langle\boldsymbol{\nabla} \cdot\left(h^{o} \boldsymbol{\nabla} \phi\right)\right\rangle^{t o}+\varepsilon^{2} \boldsymbol{\nabla} \cdot\left[\left(h^{m o}+h^{m s}\right) \boldsymbol{\nabla} \phi+h^{o}\left(\boldsymbol{u}^{m o}\right.\right. \\
& \left.\left.+\boldsymbol{u}^{m s}\right)+\left(h^{o}\right)^{2} \partial_{z} \boldsymbol{\nabla} \phi / 2\right]=\gamma \int^{t} \boldsymbol{\nabla}^{2}\left(\partial_{z} \phi\right)
\end{aligned}
$$

$$
\begin{aligned}
& \partial_{t} \phi+\varepsilon\left\langle h^{\prime \prime} \partial_{t z}^{2} \phi\right\rangle^{t \prime \prime}+\varepsilon^{2}\left[\left(h^{m i s}+h^{m s}\right) \partial_{t z}^{2} \phi-h^{\prime \prime} \partial_{z} q^{m s}\right. \\
& \left.+\left(h^{\prime \prime}\right)^{2} \partial_{t z z}^{3} \phi / 2\right]+\varepsilon\left\langle|\nabla \phi|^{2}+\left|\partial_{z} \phi\right|^{2}\right\rangle^{\prime \prime \prime} / 2+\varepsilon^{2}\left[\left(\boldsymbol{u}^{m o}\right.\right. \\
& \left.\left.+\boldsymbol{u}^{m i x}\right) \cdot \nabla \phi+h^{\prime \prime} \partial_{z}\left(|\boldsymbol{\nabla} \phi|^{2}+\left|\partial_{z} \phi\right|^{2}\right) / 2\right]+4 a\left\langle h^{o} \sin 2 t\right\rangle^{t o} \\
& +(1-\Gamma) h^{o}-\Gamma \boldsymbol{\nabla} \cdot\left[\boldsymbol{\nabla} h^{\prime \prime} /\left(1+\varepsilon^{2}\left|\nabla h^{\prime \prime}\right|^{2}\right)^{1 / 2}\right]+\gamma \partial_{z z}^{2} \phi=0,
\end{aligned}
$$

$$
w^{n o}=0, \quad d_{z} \boldsymbol{u}^{m o}=2\left\langle\left\langle\left(\boldsymbol{\nabla} h^{o} \cdot \boldsymbol{\nabla}\right) \boldsymbol{\nabla} \phi+\left(\nabla^{2} \phi\right) \nabla h^{o}\right\rangle^{t s}\right\rangle^{h o},
$$

$$
\partial_{z} \boldsymbol{u}^{m x}=2\left\langle\left\langle\left(\nabla h^{\prime \prime} \cdot \nabla\right) \nabla \phi+\left(\nabla^{2} \phi\right) \nabla h^{o}\right\rangle^{h s}\right\rangle^{h s},
$$

$$
q^{n s s}-(1-\Gamma) h^{m s s}=0,
$$

$$
\partial_{i} h^{m s s}+\nabla \cdot\left(\int_{d}^{0} \boldsymbol{u}^{m s} d z\right)=-\nabla \cdot\left(\left\langle\left\langle h^{\prime} \nabla \phi\right\rangle^{h s}\right\rangle^{h s}\right)
$$

$$
-(\mathbf{1}-\mathbf{\Gamma}) h^{m(\prime)}+\mathbf{I} \nabla^{2} h^{m o}=\left\langle\left\langle h^{\prime \prime} \partial_{t z}^{2} \phi+\left(|\nabla \phi|^{2}+\left|\partial_{z} \phi\right|^{2}\right) / 2\right\rangle^{\prime s}\right\rangle^{h o} \text {. }
$$

at $z=0$; and periodic boundary conditions in the horizontal direction as in Eqs. (69) and (75). 
This linear approximation exactly provides the first bifurcated branch of standing waves (SWs) from the planar base state with associated mean flow that is unforced [sec Eq. (56)] and thus identically vanishes at large times. This approximation is also exact for the lincar stability of the SWs and, in particular, for the instability threshold of this branch, namely the threshold amplitude (if it is finite) for the appearance of transverse amplitude modulations (TAMs) [5]. Furthermore, we expect that the neglected convective terms do not play a significant qualitative role in subsequent bifurcated branches of TAMs, at least near threshold. In addition, this approximation is almost exact for stripes because convective terms can be neglected in this case, as already explained in Sec. III A.

\section{Two-dimensional approximation}

We introduce next a (drastic) single-mode approximation for the $z$ dependence of the mean flow variables in Eqs. (88)-(98). We write horizontal velocities as

$$
\begin{gathered}
\boldsymbol{u}^{m o}=g(z) \boldsymbol{U}^{m o}, \quad q^{m o}=g(z) Q^{m o}, \quad \boldsymbol{u}^{m s}=g(z) \boldsymbol{U}^{m s}, \\
q^{m o}=g(z) Q^{m s s},
\end{gathered}
$$

where the function $g$ is such that

$$
\begin{gathered}
g(-d)=g^{\prime}(0)=0, \quad \int_{d}^{0} g(z)^{2} d z=1, \\
g(0)>0, \quad \int_{-d}^{0} g(z) d z>0 .
\end{gathered}
$$

This function is otherwise arbitrary and can be selected to yield the best approximation to the vertical velocity profiles. A reasonable choice is

$$
g(z)=\sqrt{2 / d} \sin [\pi(z+d) /(2 d)]
$$

which satisfies

$$
g^{\prime \prime}=-\lambda g, \quad \text { with } \lambda=\frac{\pi^{2}}{4 d^{2}} .
$$

By using this simplification, Eqs. (89), (90), (92), and (98) reduce 10

$$
\begin{aligned}
& \nabla \cdot \boldsymbol{U}^{m o}=0 \\
& \partial_{f} \boldsymbol{U}^{m \omega}=-\varepsilon^{2} \nabla Q^{m o}+\gamma\left(\nabla^{2} \boldsymbol{U}^{m i}-\lambda \boldsymbol{U}^{m i}\right) / 2 \\
& +\beta_{1} \gamma\left[\left\langle\left\langle\left(\nabla h^{o} \cdot \nabla\right) \nabla \phi+\left(\nabla^{2} \phi\right) \nabla h^{o}\right\rangle^{t s}\right\rangle^{h o}\right]_{z-0},
\end{aligned}
$$

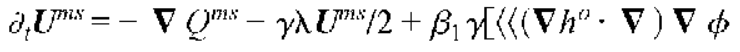

$$
\begin{aligned}
& \left.\left.\left.+\left(\nabla^{2} \phi\right) \nabla h^{o}\right\rangle^{t s}\right\rangle^{h s}\right]_{z-0} \\
& \beta_{1} Q^{m s}-(1-\Gamma) h^{m s}=0 \\
& \partial h^{m x}+\beta_{2} \boldsymbol{\nabla} \cdot \boldsymbol{U}^{m s}+\boldsymbol{\nabla} \cdot\left(\left\langle\left\langle h^{o} \boldsymbol{\nabla} \phi\right\rangle^{\langle s}\right\rangle^{h w}\right)_{z^{-0}}=0
\end{aligned}
$$

with

$$
\beta_{1}=g(0)>0, \beta_{2}=\int_{-d}^{0} g(z) d z>0
$$

Note that if $\mathrm{g}$ is given by Eq. (102), then

$$
\beta_{1}=\sqrt{2 / d}, \quad \beta_{2}=\sqrt{8 d} / \pi
$$

The complete set of equations also includes Eq. (62) and the following equations and boundary conditions which follow from Eqs. (88), (94), and (95):

$$
\nabla^{2} \phi+\partial_{z \Sigma}^{2} \phi+\varepsilon^{2} g^{\prime \prime}(z)\left(\iint^{l} \int \phi\right) \cdot\left(\boldsymbol{U}^{m o}+\boldsymbol{U}^{m s}\right)=0
$$

in $-d<z<0$, with $\partial_{z} \phi=0$ at $z=-d$, and

$$
\begin{aligned}
& \partial_{t} h^{o}-\partial_{z} \phi+c\left\langle\boldsymbol{\nabla} \cdot\left(h^{\circ} \boldsymbol{\nabla} \phi\right)\right\rangle^{t o}+\varepsilon^{2} \boldsymbol{\nabla} \cdot\left[\left(h^{m o}+h^{m s}\right) \boldsymbol{\nabla} \phi\right. \\
& \left.+\beta_{1} h^{o}\left(\boldsymbol{U}^{m o}+\boldsymbol{U}^{m s}\right)\right]+\varepsilon^{2} \nabla \cdot\left[\left(h^{o}\right)^{2} \partial_{z} \nabla \phi / 2\right] \\
& =\gamma \int^{t} \nabla^{2}\left(\partial_{z} \phi\right) \text {. } \\
& \partial_{t} \phi+\varepsilon\left\langle h^{o} \partial_{t z}^{2} \phi\right\rangle^{t o}+\varepsilon^{2}\left[\left(h^{m o}+h^{m s}\right) \partial_{t z}^{2} \phi+\left(h^{o}\right)^{2} \partial_{t z z}^{3} \phi / 2\right] \\
& +\varepsilon\left\langle|\nabla \phi|^{2}+\left|\partial_{z} \phi\right|^{2}\right\rangle^{t o} / 2+\varepsilon^{2}\left[\beta_{1}\left(\boldsymbol{U}^{n o}+\boldsymbol{U}^{m s}\right) \cdot \boldsymbol{\nabla} \phi\right. \\
& \left.+h^{\prime} \partial_{z}\left(|\nabla \phi|^{2}+\left|\partial_{z} \phi\right|^{2}\right) / 2\right]+4 a\left\langle h^{\circ} \sin 2 t\right\rangle^{\prime \prime}+(1-I) h^{\circ} \\
& -\Gamma \boldsymbol{\nabla} \cdot\left[\boldsymbol{\nabla} h^{\prime \prime} /\left(1+\varepsilon^{2}\left|\nabla h^{\prime \prime}\right|^{2}\right)^{1 / 2}\right]+\gamma \partial_{z z}^{2} \phi=0,
\end{aligned}
$$

at $z=0$. Equations (62) and (104)-(112) must be integrated with periodic boundary conditions in the horizontal directions, as above. Note that the mean flow does not contributc to the averaged encrgy equation at leading order [sec Eq. (35)].

\section{A phenomenological description}

We finally discuss the simplest possible approximation to this problem by considering a phenomenological model of Faraday waves that qualitatively describes its primary bifurcation and secondary instabilitics [33]. It involves a complex order parameter $\psi$ that satisfies

$$
\partial_{i} \psi=-\gamma \psi+i f \bar{\psi}+3 i\left(1+\nabla^{2}\right) \psi / 4+(i-\gamma \alpha)|\psi|^{2} \psi
$$

A derivation of this equation is given in the Appendix. The order parameter $\psi$ is a linear combination of the free surface deflection and (a vertical average of) the velocity potential, 


$$
\begin{aligned}
h^{o}= & \psi e^{-i \omega t}+\mathrm{c} \cdot \mathrm{c} ., \phi=-i \psi e^{-i \omega t}+\mathrm{c} . \mathrm{c} ., \text { or } \psi=e^{-i \omega t}\left(h^{o}\right. \\
& +i \phi) / 2
\end{aligned}
$$

Despite its simplicity, the order parameter model qualitatively describes some of the features of the Faraday instability: The linear dispersion relation coincides with that of the fluid in the limit of low viscous damping, and the model exhibits a primary bifurcation to a standing-wave solution near threshold, which can be either sub- or supercritical at threshold depending on the wave number. Also, stationary solutions are in turn destabilized against amplitude and phase modulation instabilities. For sufficiently high supercriticalities, the solutions of Eq. (113) exhibit spatiotemporal chaos. Consistent with the weakly dissipative limit we are considcring in this paper, we must assume that

$$
\gamma \ll 1, f \ll 1,|\alpha| \sim 1 .
$$

This model has already been used by Kiyashko et al. [34] to understand how mean flow effects might induce rotating patterns in a Faraday wave experiment. Conjecturing that rotation was somehow due to the mean flow produced by surface waves, they added a convective term $-\boldsymbol{u} . \nabla \psi$ to the right-hand side of Eq. (113), where $\boldsymbol{u}$ was a velocity field that was given independently of $\%$, thus ignoring any coupling between surface waves and mean flow.

Here we shall add a similar term to the right-hand side of Eq. (113) but, given the analysis above, $\boldsymbol{u}$ evolves with the surface waves according to a phenomenological equation with the appropriate symmetries. First we replace Eq. (113) with

$$
\begin{aligned}
\partial_{t} \psi= & -\gamma \psi \psi+i f \bar{\psi}+3 i\left(1+\nabla^{2}\right) \psi / \psi / 4+(i-\gamma \alpha)|\psi|^{2} \psi-\beta_{1}\left(\boldsymbol{U}^{m o}\right. \\
& \left.+\boldsymbol{U}^{m u s}\right) \cdot \boldsymbol{\nabla} \psi
\end{aligned}
$$

as suggested by Eqs. (111)-(114). Note that we are not including any dependence on $h^{m o}$ and $h^{m s}$, because this is beyond the scope of this phenomenological model. The coupling term is not conservative for general initial conditions, which is not optimal. However, this term may be seen to lead to a conservative contribution at leading order for solutions that are linear combinations of plane waves [as in Eq. (46) above]. Since $\%$ is intended here to only model the spatially oscillatory part of the llow, we require that

$$
\langle\psi\rangle^{(t)}=0
$$

The contribution from the mean flow appears through $U^{\text {no }}$ and $U^{\text {nhs }}$, which are the short- and long-wave components of the mean flow defined above. Their evolution is given by Eqs. (104)-(107) but replacing $h^{\circ}$ and $\phi$ by $\psi$ according to Eqs. (113) and (114).

$$
\begin{aligned}
& \boldsymbol{\nabla} \cdot \boldsymbol{U}^{n o}=0, \\
& \partial_{t} U^{m o}=-\varepsilon^{2} \nabla Q^{m o}+\gamma\left(\nabla^{2} \boldsymbol{U}^{m(s}-\lambda \boldsymbol{U}^{m(0)}\right) / 2 \\
& +\beta_{1}\left\langle i(\boldsymbol{\nabla} \psi \cdot \boldsymbol{\nabla}) \boldsymbol{\nabla} \bar{\psi}+i\left(\boldsymbol{\nabla}^{2} \overline{\psi k}\right) \boldsymbol{\nabla} \psi+\text { c.c. }\right\rangle^{h o} \text {, }
\end{aligned}
$$

$$
\begin{aligned}
& \partial_{t} \boldsymbol{U}^{m w s}=-\nabla Q^{m s s}-\gamma \lambda \boldsymbol{U}^{m s} / 2+\beta_{1}\langle\langle(\nabla \psi \cdot \nabla) \nabla \bar{\psi} \\
& \left.+i\left(\nabla^{2} \bar{\psi}\right) \nabla \psi+\text { c.c. }\right\rangle^{h s} \text {. } \\
& \beta_{2} Q^{m s}-(1-\Gamma) h^{m s}=0 \\
& \left.\partial_{l} h^{m i s}+\beta_{2} \nabla \cdot \boldsymbol{U}^{m i s}+\boldsymbol{\nabla} \cdot(i\langle\psi\rangle \boldsymbol{\nabla} \psi\rangle^{h s}+\text { c.c. }\right)=0 .
\end{aligned}
$$

We also require that [cf. Eqs. (103) and (108)]

$$
\beta_{1}^{4} / \lambda=16 / \pi^{2}, \quad \beta_{1} \beta_{2}=4 / \pi
$$

Equations (119)-(121) imply that $\mathbf{U}^{\mathrm{mo}}=\mathbf{U}^{\mathrm{ms}}=0$ as $\beta_{1} \rightarrow 0$. Note also that the mean flow is unforced if the surface waves are standing (the phase of $\psi$ is independent of position) as all forcing terms in Eqs. (119)-(121) vanish.

\section{SECONDARY INSTABILITIES OF THE PHENOMENOLOGICAL MODEL}

In order to obtain a qualitative picture of the effects of mean flows on surface waves, we sludy in this section secondary instabilitics of the base periodic solution of the order parameter model defined by the coupled Eqs. (113) and (118)-(121) of Sec. III D. Generally speaking, we find that mean llows couple weakly to transverse phase modulations and hence do not appreciably modify the zigzag boundary. Transverse amplitude modulations are affected by mean flows, the latter generally being destabilizing. Mean flows also increase the region of instability against longitudinal perturbations (Eckhaus) and, more importantly, introduce a finite wave-number longitudinal instability which for certain values of the parameters can render much of the parameter space in which periodic solutions exist unstable. This instability branch is of an oscillatory nature, and arises at the merging point between the branch that corresponds to longwavelength longitudinal modes of $\psi$ and a hydrodynamic branch which is weakly damped as $k \rightarrow 0$.

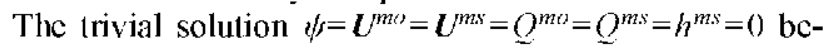
comes lincarly unstable against a periodic perturbation of $\psi$ of wave number $q$ for $\mu>\mu_{c}(q)=\sqrt{1+\left[3\left(1-q^{2}\right) / 4 \gamma\right]^{2}}-1$, the neutral stability curve. $\mu$ is the control parameter defined as $\mu=(f-\gamma) / \gamma$. The critical mode $q=1$ becomes unstable at $\mu=0$.

For small $\mu>0$, stationary and periodic solutions exist that can be approximated by a single Fourier mode $\psi_{q}(x)$ $=\alpha_{q} \cos (q x) \exp \left(i \Theta_{q}\right)$ with 


$$
\alpha_{q}^{2}=\frac{q^{2}-1-\frac{4}{3} \alpha \gamma^{2} \pm \frac{1}{3} \sqrt{16 f^{2}\left(1+\alpha^{2} \gamma^{2}\right)-\left[4 \gamma+3 \alpha \gamma\left(q^{2}-1\right)\right]^{2}}}{1+(\alpha \gamma)^{2}},
$$

where the \pm sign stands for $\operatorname{sgn}\left(1-q^{2}+4 \alpha \gamma^{2} / 3\right)$, and $\Theta_{4}$ satisfics $\sin 2 \Theta_{q}=\left(1+3 \alpha \alpha_{q}^{2} / 4\right) \gamma / f, \cos 2 \Theta_{q}=\frac{3}{4}\left(q^{2}-1-\alpha_{q}^{2}\right) / \delta$. Note that the bifurcation at threshold is subcritical if $q^{2}>1$ $+3 \alpha \gamma^{2} / 4 \simeq 1$ (recall that $\gamma$ is small) and supercritical otherwise. This solution for the order parameter leads to vanishing driving terms in the mean flow Eqs. (118)-(121); hence all mean flow variables remain zero for the base, periodic solution.

In order to address the stability of the stationary solution (123) against longitudinal perturbations, we introduce

$$
\begin{aligned}
\psi= & A_{0}\left\{\exp (i q x)+\exp (-i q x)+a^{+-} \exp [i(q+k) x]\right. \\
& +a^{+-} \exp [i(q-k) x]+a^{-+} \exp [i(k-q) x] \\
& \left.+a^{--} \operatorname{cxp}[-i(q+k) x]\right\}
\end{aligned}
$$

[where $A_{0}=\frac{1}{2} \alpha_{4} \exp \left(\mathrm{i} \Theta_{4}\right)$ ], together with the corresponding perturbations of the mean flow variables

$$
\begin{aligned}
& l_{x}^{m s}=u^{\prime} \exp (\mathrm{i} k x)+c . c . \\
& h^{m x}=c^{\prime} \exp (\mathrm{i} k x)+c . c .
\end{aligned}
$$

and

$$
Q^{m s}=d^{\prime} \exp (\mathrm{i} k x)+\text { c.c. }
$$

where $U^{m o}=0$ as seen from the incompressibility condition (118), and the definition of $\boldsymbol{u}^{\text {mo }}=0(60)$, which require that $U_{x}^{m o}=0$ and $\left\langle\boldsymbol{U}^{m o}\right\rangle^{h s}=0$, respectively.

By inserting Eq. (124) into the nonlinear terms of Eqs. (120) and (121),

$$
\begin{gathered}
\left.\mathbf{N}^{w}=i(\boldsymbol{\nabla} \psi \cdot \boldsymbol{\nabla}) \boldsymbol{\nabla} \psi+i\left(\boldsymbol{\nabla}^{2}\right\} /\right) \nabla \psi+\text { c.c. }, \\
\mathbf{N}^{i}=i \psi \boldsymbol{\nabla} \psi+\text { c.c. },
\end{gathered}
$$

and retaining only the long-wavelength components, we obtain

$$
\begin{aligned}
\left\langle N_{x}^{v^{\prime}}\right\rangle^{h s}= & \left\langle 2 i \partial_{x} \psi \sigma_{x}^{2} \bar{\psi}+c_{-} c\right\rangle^{h s}=2\left|A_{0}\right|^{2}\left\{\left[q+a^{\prime}(q+k) e^{i k x}\right.\right. \\
& \left.+a^{\prime}(q-k) e^{i k x}\right]\left[q^{2}+\bar{a}^{\prime}(q+k)^{2} e^{i k x}+\bar{a}(q\right. \\
& \left.-k)^{2} e^{i k x}\right]+\left[-q+a{ }^{\prime}(k-q) e^{i k x}-a \quad(q+k) e^{i k x}\right]\left[q^{2}\right. \\
& \left.\left.+\bar{a}^{-+}(k-q)^{2} e^{-i k x}+\bar{a}^{-}(q+k)^{2} e^{i k x}\right]\right\}+ \text { c.c. }, \quad(130) \\
\left\langle N_{x}^{i}\right\rangle^{h x}= & \left\langle i \psi \partial_{x} \bar{\psi}+\text { c.c. }\right\rangle^{h s}=\left|A_{0}\right|^{2}\left\{\left(1+a^{\cdot} e^{i k x}+a^{\prime} e^{i k x}\right)[q\right. \\
& \left.+\bar{a}^{\top} \cdot(q+k) e^{i k x}+\bar{a}^{\top}(q-k) e^{i k x}\right]+\left(1+a^{\prime} e^{i k x}\right. \\
& \left.\left.+a^{-} e^{-i k x}\right)\left[-q+\bar{a}^{--}(k-q) e^{-i k x}-\bar{a}^{-}(q+k) e^{i k x}\right]\right\} \\
& + \text { c.c. },
\end{aligned}
$$

respectively, where $N_{x}^{(v, i)}$ denote the $x$-components of the nonlincar terms $\mathbf{N}^{(w, i)}$. Equations for the perturbation ampliludes $u, c^{\prime}$, and $d^{\prime}$ are derived by linearizing Eqs. (120) and (121) with respect to all the perturbation amplitudes, after substitution of Eqs. (130) and (131), and by extracting those terms that are proportional to $e^{i k x}$ :

$$
\begin{gathered}
\partial_{t} u^{\prime}=-i k d^{\cdot}-\frac{\gamma \lambda}{2} u^{\cdot}+2 \beta_{1} \gamma\left|A_{0}\right|^{2}\left[(q+k)\left(2 q^{2}+q k\right)\right. \\
\left.\times\left(a^{\prime}-\bar{a}\right)+(q-k)\left(2 q^{2}-q k\right)\left(\overline{a^{\prime}}-a^{\prime}\right)\right] \\
\beta_{2} d^{\cdot}=(1-\Gamma) c^{\prime} \\
\partial_{c^{\prime}} c^{+}=-\beta_{2} i k u^{+}-i k\left|\Lambda_{1}\right|^{2}\left[(2 q+k)\left(a^{-+}-\bar{a}^{--}\right)\right. \\
\left.+(2 q-k)\left(\bar{a}^{+-}-a^{-+}\right)\right] .
\end{gathered}
$$

Finally, the governing equations for the perturbation amplitudes of the order parameter are obtained by linearizing Eq. (116) and extracting the amplitudes of the Fourier modes $\exp i( \pm q \pm k) x$. For example, the governing equation for $a^{+-}$ is

$$
\begin{aligned}
\partial_{t} a^{+-}= & -\gamma a^{+-}+i f \frac{\overline{A_{0}}}{A_{0}} \overline{a^{--}}+\frac{3 i}{4}\left[1-(q+k)^{2}\right] a^{+-}+(i-\alpha \gamma) \\
& \times\left|A_{0}\right|^{2}\left(4 a^{\cdot}+2 a+a^{\prime}+2 a^{\prime}\right)-\beta_{1} i q u^{\prime} .
\end{aligned}
$$

Similar equations result for the other three amplitudes.

We now have a system of six first-order ordinary differential equations which is linear in the perturbation amplitudes $a^{ \pm x}, u^{+}$, and $c^{+}$. The matrix of right-hand side coefficients is denoted by $A(q, k, \varepsilon, \ldots)$, and is a function of the wave numbers of the base solution $q$ and of the perturbation $k$, of the control parameter $\epsilon$, and of the other parameters of the model. The base solution becomes unstable when the real part of any eigenvalue of this matrix becomes positive. We have numerically obtained the eigenvalues of the matrix $A$, and determined the region of stability of the base solution. Two types of instabilitics are possible: a standard longwavelength Eckhaus instability which depends on the mean flow, and a finite wave-number oscillatory instability, which is completely due to the mean flow. As was the case in the asymptotically exact equations for one-dimensional Faraday waves [12], this latter instability only occurs with nonzero mean flow. Both instabilities will be discussed further below.

The stability of periodic solutions against transverse amplitude and phase perturbations can be studied in a similar fashion. Given that $\partial_{y} \psi_{q}=0$ in the base state with zero mean flow, terms involving the $y$ components of the mean flow will be of second order in the amplitudes of the perturbation and hence only the components $U_{x}^{\text {mio }}$ and $U_{x}^{m s s}$ need to be 
perturbed. However, and in contrast to the case of a longitudinal perturbation, both short- and long-wavelength components of the mean flow need to be included. Furthermore, the equations for $U_{x}^{\text {tho }}$ and $U_{x}^{n t s}$ decouple at linear order, and they can be analyzed separately. We show next that only the $U_{x}^{\text {mo }}$ part modifies the transverse amplitude modulation (TAM) instability line, whereas the zigzag line is not affected by either component.

We start by considering the short-wavelength component of the mean flow velocity $U_{x}^{m o}$, and introduce the following perturbation for the order parameter:

$$
\begin{aligned}
\psi= & A_{0}\left\{\exp (i q x)+\exp (-i q x)+a^{\cdot} \exp [i(q x+k y)]\right. \\
& +a \exp [i(q x-k y)]+a \cdot \exp [i(-q x+k y)] \\
& +a \exp [-i(q x+k y)]\}, \\
l_{x}^{\prime n o}= & v^{++} \operatorname{cxp}[i(2 q x+k y)]+v^{+-} \operatorname{cxp}[i(2 q x-k y)]+\text { c.c. },
\end{aligned}
$$

and

$$
Q^{m o v}=p^{\prime} \exp [i(2 q x+k y)]+p^{\prime} \exp [i(2 q x-k y)]+c . c .
$$

The amplitude of the mode $e^{i 2 q x}$ in the $x$ component of the nonlinear forcing term of Eq. (119) is

$$
\begin{aligned}
& \left\langle N_{x}^{\prime \prime}\right\rangle^{h o}=\left\langle\left[\partial_{x} \psi \psi \partial_{x}^{2} \bar{\psi}+\partial_{y} \psi \gamma \partial_{x y} \bar{\psi} \psi\left(\partial_{x}^{2} \bar{\psi}+\partial_{y}^{2} \bar{\psi} \bar{\gamma}\right) \partial_{x} \psi\right]+\text { c.c. }\right\rangle^{h o} \\
& =\left|A_{0}\right|^{2} q k^{2}\left[\left(\bar{a} \cdot-a^{\prime}\right) e^{i k y}+\left(\bar{a}-a^{\prime \prime}\right) e^{i k y}\right] e^{i 2 \psi x}+\text { c.c. }
\end{aligned}
$$

where in Eq. (140) only terms linear in $a^{\perp \perp}$ and $\overline{a^{\perp-}}$ have been kept. The pressure $Q^{m e}$ is calculated by taking the divergence of Eq. (119),

$$
0=-\nabla^{2} Q^{n o}+\beta_{1} \gamma\left(\partial_{x} N_{x}^{l i}+\partial_{y} N_{y}^{\prime \prime}\right)
$$

and is thus climinated by using

$$
0=\left(4 q^{2}+k^{2}\right) p^{-+}+\beta_{1} \gamma i 2 q\left|A_{0}\right|^{2} q k^{2}\left(\overline{a^{--}}-a^{++}\right),
$$

and a similar equation for $p^{--}$(here we have used the fact that one obtains $\left\langle\lambda_{y}^{n}\right\rangle^{h o}=0$ at linear order). We then derive the following linear system of equations for the perturbation amplitudes:

$$
\begin{aligned}
\partial_{t} a^{++}= & -\gamma a^{++}+i f \frac{\overline{A_{0}}}{A_{0}} a^{--}+\frac{3 i}{4}\left(1-q^{2}-k^{2}\right) a^{+-}+(i-\alpha \gamma) \\
& \times\left|A_{0}\right|^{2}\left(4 a^{\prime}+2 \bar{a}+\bar{a}^{\prime}+2 a^{\prime}\right)+\beta_{1} i q v^{\cdot 1},(143) \\
\partial_{t} v^{+-}=- & \frac{\gamma}{2}\left(4 q^{2}+k^{2}+\lambda\right) v^{++}+\beta_{1} \gamma\left|A_{0}\right|^{2} \frac{q k^{4}}{4 q^{2}+k^{2}}\left(\bar{a}^{--}-a^{+-}\right),
\end{aligned}
$$

with similar equations for $a^{--}, a^{-+}, a^{+-}$, and $v^{+-}$.

A TAM is defined by the linear combinations $b_{1}=a^{+-}$ $+a^{+-}+a^{-+}+a^{--}$and $v_{1}=\operatorname{Im}\left(v^{-+}+v^{+-}\right)$. From Eqs. (143) and (144), we find a closed system

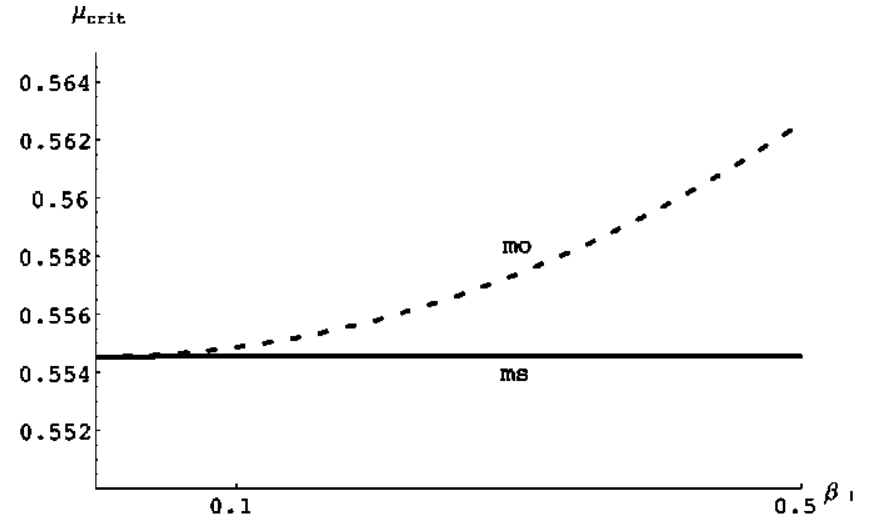

FIG. 1. This figure illustrates the weak destabilization of the base solution against transverse amplitude modulations due to the coupling to mean flows. Shown are the critical values for instability $\mu_{\text {crit }}$ arising from either the long-wavelength component of the mean flow velocity alone ("nns") or the shorl-wavelength component alone ("mo") as a linction of the coupling parameter $\beta_{1}$. The base solution is stable above the corresponding lines. The figure shows that the "ms" component of the flow does not appreciably modify the stability threshold, whereas the effect of the "mo" component is to weakly destabilize the base solution with increasing $\beta_{1}$. The wave number of the base solution is $q^{-1.04}$, and we have used $\gamma=0.1, \alpha=0.5$, and $\Gamma=0.8$. The parameter $\beta_{1}$ spans the range between no mean flow and the approximate value that corresponds to the weak viscosity but shallow layer experiments of Rel. [5]. The values of $\beta_{2}$ and $\lambda$ depend on $\beta_{1}$ according to $\mathrm{Eq}$. (122).

$$
\begin{aligned}
\partial_{1} b_{1}= & -\gamma b_{1}+i f \frac{\overline{A_{0}}}{A_{0}} \bar{b}_{1}+\frac{3 i}{4}\left(1-q^{2}-k^{2}\right) b_{1} \\
& +\frac{3}{4} \alpha_{q}^{2}(i-\alpha \gamma)\left(2 b_{1}+b_{1}\right)-2 \beta_{1} q v_{1},
\end{aligned}
$$

$$
\partial_{t} v_{1}=-\frac{\gamma}{2}\left(4 q^{2}+k^{2}+\lambda\right) v_{1}-\frac{\beta_{1} \gamma}{4} \alpha \alpha_{q}^{2} \frac{q k^{4}}{4 q^{2}+k^{2}} \operatorname{Im} b_{1}
$$

The relevance of mean flows on this perturbation is demonstrated in Fig. 1, showing the stability boundary in the plane $\left(\beta_{1}, \mu\right)$ at fixed wave number $q$ and model parameters. With increasing mean flow coupling $\beta_{1}$, the region of stability of the base solution (region above the dashed line in the figure) decreases.

Altcrnatively, a transverse phase modulation (rig/ag) is given by $b_{2}=a^{\prime \prime}-a^{\prime}-a^{\prime}+a$ and $v_{2}=\operatorname{lm}\left(v^{\prime}-v^{\prime}\right)$. We find in this case

$$
\begin{aligned}
\partial_{t} b_{2}= & -\gamma b_{2}+i f \frac{A_{0}}{A_{0}} \overline{b_{2}}+\frac{3 i}{4}\left(1-q^{2}-k^{2}\right) b_{2} \\
& +\frac{1}{4} \alpha_{q}^{2}(i-\alpha \gamma)\left(2 b_{2}+\overline{b_{2}}\right)-2 \beta_{1} q v_{2},
\end{aligned}
$$




$$
\partial_{t} v_{2}=-\frac{\gamma}{2}\left(4 q^{2}+k^{2}+\lambda\right) v_{2}-\frac{\beta_{1} \gamma}{4} \alpha_{q}^{2} \frac{q k^{4}}{4 q^{2}+k^{2}} \operatorname{Im} b_{2} .
$$

From the eigenvalue equation of this system, it can be shown that modifications to the eigenvalues due to $v_{2}$ appear at higher order in $k$. Since the zigzag instability occurs in the limit $k \rightarrow 0$, we find the effect of mean flow to be negligible in this case.

A similar analysis has been performed for the longwavelength component of the mean flow with perturbations of the form $U_{x}^{m s}=u^{\prime} \exp (\mathrm{i} k y)+\mathrm{c} . c ., h^{m s}=c \cdot \exp (\mathrm{i} k y)+\mathrm{c.c}$., and $Q^{m i s}=d \exp (\mathrm{i} k y)+c . c$. The order parameter is again given by Eq. (136). As shown in Fig. 1, the contribution of the longwavelength component of the mean flow to transverse modulations is negligible.

We now turn to a summary of our numerical results about the base periodic solution. Figure 2 shows the various stability boundaries for the special cases of $\beta_{1}=0$ (no mean flow) and $\beta_{1}=0.5$. Other values of the parameters used are $\gamma$ $=0.1, \alpha=0.5$, and $\Gamma=0.8$. The values of $\beta_{2}$ and $\lambda$ depend on $\beta_{1}$ according to Eq. (122). Except for $\alpha$, these parameter values correspond approximately to the values for the lowviscosity experiments of Kudrolli and Gollub described in [5]. For instance, typical experimental values of $\beta_{1}=\sqrt{2 / d}$ (where $d$ is the dimensionless height of the layer) are between 0.5 and 1.2 . Figure 2 includes the neutral stability curve of the basic periodic solution and, since the primary bifurcation is subcritical for $q>1$, we have included the saddle-node curve where the periodic solution bifurcates (a subcritical bifurcation for $q>1$ as $\gamma \rightarrow 0$ has also been found in a direct numerical solution of the governing lluid cquations in two dimensions [35]). The case $\beta_{1}=0$ is shown as a reference, and it agrees with the results of [33].

The range of base solutions that is stable against all perturbations considered here (Eckhaus, TAM, and zigzag) is a small region close to threshold at $\mu=0$ between the TAM and zigzag lines. Periodic solutions are stable against transverse perturbations below the dashed-dotted line in the figure (zigZag, denoted " $Z$ "), and above the dashed line (TAM). Eckhaus perturbations have a negative growth rate below the dotted line. We observe that with increasing $\beta_{1}$, both Eckhaus and TAM curves are shifted so that larger regions in the $(\mu, q)$ space become destabilized with respect to TAM or Eckhaus perturbations. As discussed above, the zigzag line is not afrecied by the mean llow.

We finally discuss a new oscillatory instability against longitudinal perturbations which is absent for $\beta_{1}=0$. The oscillatory nature of the instability is demonstrated in Fig. 3, which shows the real and imaginary parts of the corresponding critical eigenvalue branch as a function of the wave number of the perturbation $k$ for fixed $q, \mu$, and other model parameters. The imaginary part of the eigenvalue $\sigma$ is not zero at the point in which $\operatorname{Re}(\sigma)=0$. This figure also shows that the instability occurs at small but finite wave number $k$, a fact that has been confirmed by calculating the wave number with the largest growth rate both slightly above and below the instability threshold at $\mu=0.1155$.
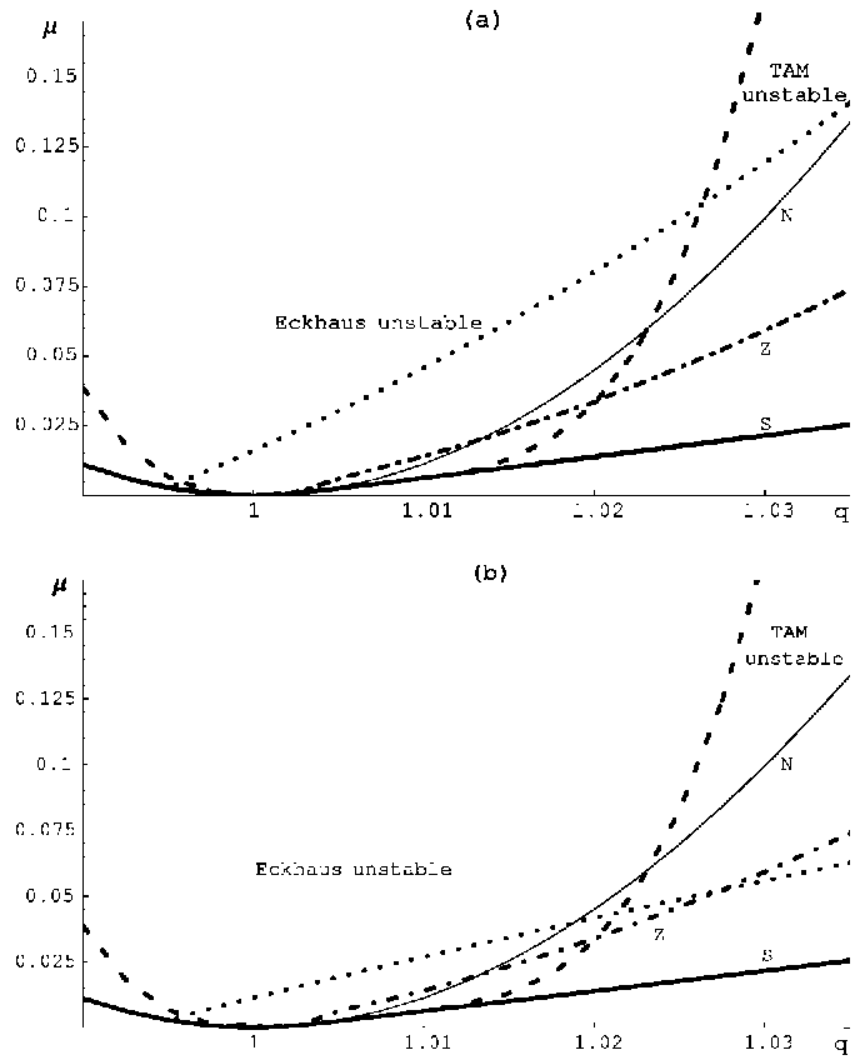

li[G. 2. Stability diagram for (a) the order parameter model of liq. (113) without mean flow, and (b) with $\beta_{1}=0.5$. Other values of the paramelers used are $\gamma-0.1, \alpha-0.5$, and $\Gamma-0.8$. We show the Eckhaus line (dotted), TAM line (dashed), and the zigzig line (dashdotted). We also show the neutral stability curve of the primary instability (solid line denoted by $N$ ), and a saddle-node bifurcation (thick solid line marked $S$ ). Only the left branch of the Eckhaus line is shown emanating from $\left(q^{-1}, \mu^{-0}\right)$. This line is parabolic near the critical point, but quickly bends to the right as shown in the figure. llence the region of stability of the base solution against an Eckhaus instability is the region below the dotted line. Comparison of (a) and (b) shows that the mean flow decreases the regions of stability against Eckhaus modulations and to a small extent against transverse amplitude modulations. In (a), the region of stability against all perturbations is shown by the gray area. This region is not indicated in (b) since these solutions are unstable with respect to the oscillatory instbility [38].

The inset in Fig. 3 illustrates the origin of the oscillatory instability. In the limit of $k \rightarrow 0$, there are two distinct eigenvalue branches that have a small and negative real part. The upper branch is marginal at $k=0$ and is related to the translational symmetry broken by the base state $\psi_{y}(x)$. On the other hand, the mean flow velocity vanishes in the base state, originating the lower branch which is weakly damped at $k$ $=0$. The damping rate of the relevant mode for longitudinal perturbations $\left(l_{x}^{m s}\right)$ is $\gamma \lambda / 2$, as can be seen from Eq. (120). As $k$ increases, the two (real) eigenvalue branches merge, leading to a complex-conjugate pair and to an oscillatory instability.

If the eigenvalue problem for the Eckhaus-type perturbations is expressed by the real and imaginary parts of the coefficients $a^{\mp \pm}, u^{+}$, and $c^{-}$, two pairs of complex-conjugate 

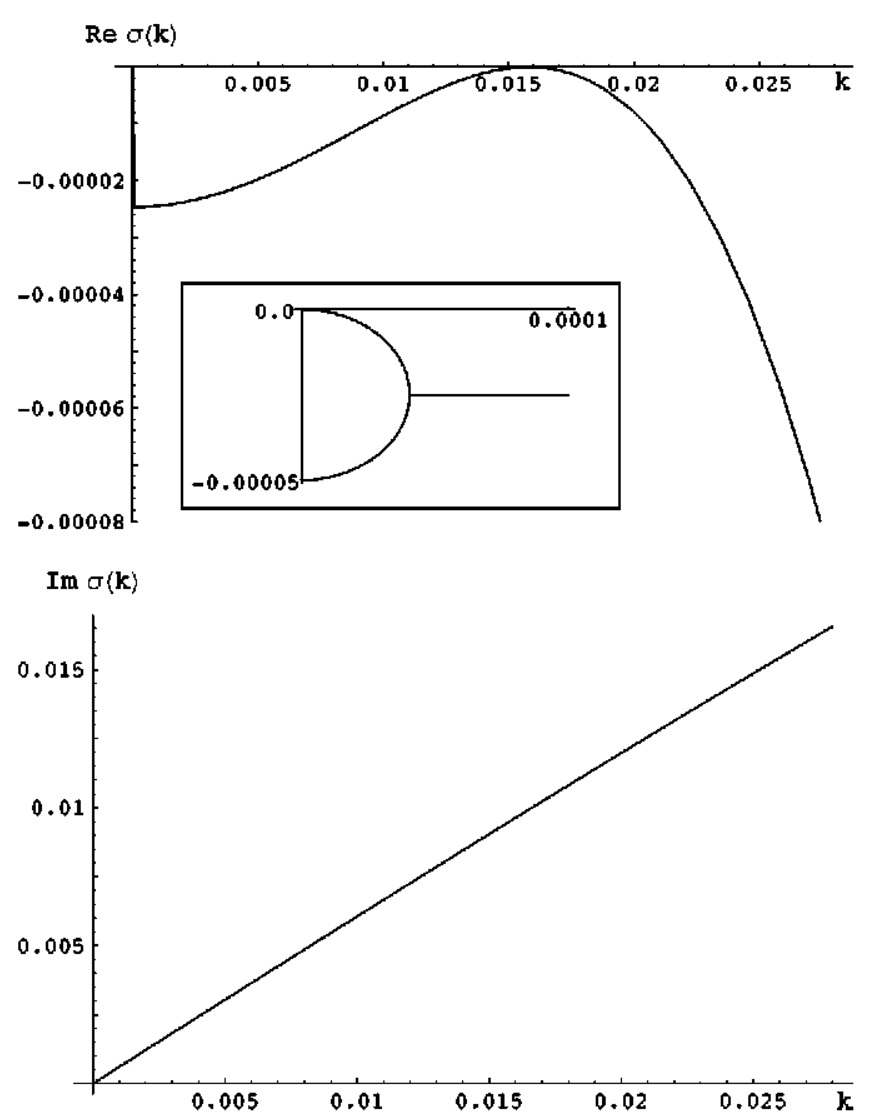

FIG. 3. Real (1op) and imaginary (bottom) parts of the largest cigenvalue for $\beta_{1}-0.2, \mu^{-} 0.1318$, and $q^{-1.07}$. The inset shows the region near $k=0$.

cigcnvalues cross the imaginary axis at the instability point. Therefore, the effective dimensionality of the critical subspace is 4 . This can be understood from the symmetry group of the system of Eqs. (132)-(136). These equations are invariant under a spatial reflection (cxchanging $a^{+-}$with $a^{--}$, $a^{--}$with $a^{-+}$, and $u^{+}$with $-\overline{u^{+}}$) and rolation [acling on $a^{-+}$and $u^{+}$as multiplication by $\exp (\mathrm{i} \Theta)$ and on $a^{--}$by $\exp (-\mathrm{i} \Theta)$, where $\Theta$ is arbitrary] that derive from the symmetry of the original equations. The corresponding symmetry group $\mathrm{O}(2)$ has two-dimensional irreducible representations, which in turn requires each eigenvalue to occur twice. At threshold, the cigenspace of the linear system is spanned by four linearly independent eigenvectors, two for each pair of the complex-conjugate cigenvalues. Symmetric bifurcation theory shows that for generic bifurcations with $O(2)$ symmetry, the nonlinear solution branches are standing and traveling waves [36]. Examples of the temporal evolution of eigenvectors of both types are plotted in Fig. 4. In practice, the specific values of the parameters determine the way the standing and traveling waves bifurcate [37]. If both are supercritical, the one with the larger amplitude would be stable, the other one unstable.

We finally show our results concerning the location of the oscillatory instability boundary as a function of $q$ for two values of $\beta_{1}$ in Fig. 5. Stable regions are located to the right of the plotted curves. As expected, the stability boundary moves toward the Eckhaus line with decreasing $\beta_{1}$, presum-
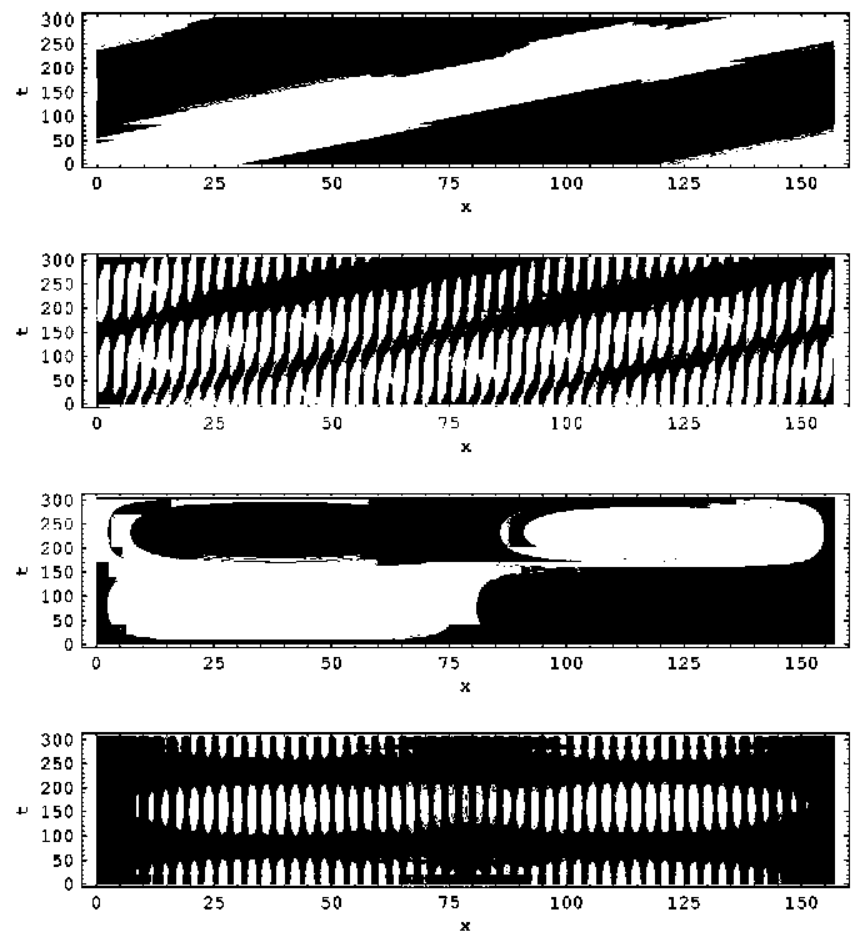

FIG. 4. Critical cigenvectors corresponding to the oscillatory instability at $\beta_{1}-0.2, q^{-1.0, \mu^{-0}}-1318$, and $k^{-0.04}$. We show the temporal evolution over its own period (vertical axis) to illustrate the nature of the critical modes. The eigenvectors are represented by the $x$ component of $u^{n s s}$ (a,c) and the amplitude of $\psi(\mathrm{b}, \mathrm{d})$. The cvolution is obtained from the two critical eigenvectors $v_{i}$ of the complex matrix $A(q, k, \ldots)$ with eigenvalues $I i(b)$ I, eft and right traveling $(\mathrm{a}, \mathrm{b})$ waves are given by the evolution of $v_{1} e^{i e g a t}$ and $v_{2} e^{-i t u t}$, respectively. A slanding wave is oblained $(\mathrm{c}, \mathrm{d})$ by superposition of the cvolution from both eigenvectors scaled to equal amplitude. The actual solution for $\psi$ is obtained from the superposition of the plotted eigenvectors with the base solution, see Eq. (124).

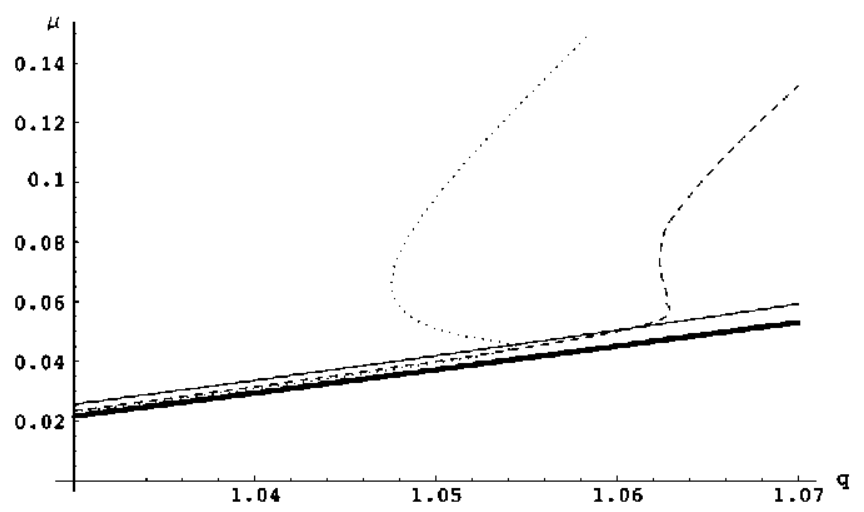

FIG. 5. Stability boundaries of the oscillatory instability for three values of $\beta_{1}: 0.05$ (dotted line), 0.2 (dashed line), and 0.5 (solid line). The thick solid line indicates the saddle node. Periodic solutions are unstable to the lelt of the eurves. Comparison with Fig. 2 shows that all periodic solutions are unstable and the pattern is expected to be time-dependent. 
ably merging with it for $\beta_{1} \rightarrow 0$. Note that for $\beta_{1}=0.5$, the unstable region covers most of the region of existence of the base states except for a narrow stripe close to the saddlcnode bifurcation.

\section{DISCUSSION}

The coupled system of equations describing fast surface oscillations and slowly evolving mean llows in three dimensions has been derived. Mean flows are forced by the surface waves by various mechanisms. For a particular choice of geometry and limit of parameters which are relevant to recent experiments, we have shown that two contributions appear at the appropriate order in the multiple scale expansion: A viscous streaming flow forced at the free surface with components of both similar and larger scale compared to the length scale of the surface waves, and a long-wavelength component originating from slow distortions of the surface elevation that exists even in the absence of viscosity.

The analysis presented has illustrated the importance of mean flows in the Faraday wave system for small viscous damping by determining the stability boundaries of the base pattern of standing waves against long-wavelength perturbations. Since the full system of surface wave/mean flow cquations is quite involved, we have instead carricd out the stability analysis of a phenomenological order parameter equation, similar in spirit to the Swift-Hohenberg model of Rayleigh-Bénard convection. In addition, we have limited the analysis to the simplest regular pattern consisting of stripes. Mean flows are induced by perturbation of the stripes, and their coupling to the order parameter equation affects the stability of stripe solutions. We have found that the mean flows generally destabilize the base solution. The strongest coupling, and hence the strongest destabilization, occurs for longitudinal or Eckhaus perturbations. Furthermore, mean flows introduce a new oscillatory instability which for small nonlinear damping in the phenomenological model renders all stripe pallerns unstable. A weaker effect has been found for finite wavelength transverse amplitude modulations which largely couple only to the short wave part of the mean llow.

Our first remark concerning experiments follows from the existence of a longitudinal oscillatory instability. Within the phenomenological model, all stripe solutions which are stable in the absence of mean flows are unstable against longitudinal oscillatory perturbations for sufficiently large coupling parameter $\beta_{1}$. One would then expect time-dependent behavior at onset. The eigenvectors corresponding to the oscillatory instability (Fig. 4) show that the associated mean flow consists of large-scale rolls with their axis oriented parallel to the surface. At the surface, it advects the waves leading to compression and dilation of waves similar to Eckhaus perturbations, but in the form of traveling or standing waves. A numerical solution of the coupled order parameter mean llow equations shows that the compression not only leads to a decrease in wave amplitude, but can also result in a complex cycle including the annilitation of stripes, possibly due to a different instability triggered by the compression. We also anticipate novel phenomena arising from mean flows if one considers the slow dynamics of defects in the wave pattern. Defects as local perturbations of a regular pattern drive mean flows, which in turn affect defect motion.

\section{ACKNOWLEDGMENTS}

This research was partially supported by the Spanish DGI under Grant No. BFM2001-2363, and by the U.S. Department of Energy under Contract No. DE-FG05-95ER 14566.

\section{APPENDIX: ORDER PARAMETER MODEL}

We briefly summarize in this appendix the derivation of the phenomenological model given by Eq. (113) of Sec. III D, first introduced in Ref. [33]. We follow the description originally introduced by Zakharov [38], and later of Crawford, Saffiman, and Yuen [39] in their sludy of the nonlinear cvolution of decp water gravity waves in an inviscid, incompressible, and irrolational fluid. Their analysis can be straightforwardly extended to a parametrically driven lluid, and linear viscous damping is added in a phenomenological way. All the variables used in this appendix are assumed to be dimensional quantities.

The governing equation for the inviscid fluid is

$$
\left(\nabla^{2}+\partial_{z}^{2}\right) \phi=0, \quad-\infty<z<h(\mathbf{x}, t),
$$

with boundary conditions at the free surface $z=h(\mathbf{x}, t)$,

$$
\begin{gathered}
\partial_{t} h+\nabla h \cdot \nabla \phi=\partial_{z} \phi, \\
\partial_{t} \phi+\frac{1}{2}(\nabla \phi)^{2}+\frac{1}{2}\left(\partial_{z} \phi\right)^{2}+\left[g_{0}+g_{z}(t)\right] h \\
=\frac{\sigma}{\rho} \nabla \cdot\left(\frac{\nabla h}{\sqrt{1+(\nabla h)^{2}}}\right),
\end{gathered}
$$

with $\sigma$ the interfacial tension and $\rho$ the density of the fluid that is being vibrated with acceleration $\left[-g_{0}-g_{z}(t)\right]$ in the $z$ direction. It is well known that this problem admits a Hamiltonian formulation with the Hamiltonian

$$
\begin{aligned}
H= & \frac{1}{2} \int d \mathbf{x} \int_{-\infty}^{h(\mathbf{x}, t)} d z\left[(\boldsymbol{\nabla} \phi)^{2}+\left(\partial_{z} \phi\right)^{2}+\frac{1}{2}\left[g_{0}+g_{z}(t)\right] h^{2}\right. \\
& \left.+\frac{\sigma}{\rho}\left[\sqrt{1+(\boldsymbol{\nabla} h)^{2}}-1\right]\right],
\end{aligned}
$$

where the velocity potential further satisfies the boundary condition $\partial_{z} \phi=0$ as $z \rightarrow-\infty$. The canonically conjugate variables are the surface deflection $h(\mathbf{x}, t)$ and the velocity poIential on the surface $\phi^{r}(\mathbf{x}, t)=\phi(\mathbf{x}, z=h(\mathbf{x}))$. Phenomenological damping can be introduced by considering a dissipation function $Q\left(h(\mathbf{x}, t), \phi^{s}(\mathbf{x}, t)\right)$. The resulting canonical equations of motion are

$$
\partial_{t} h(\mathbf{x}, t)=\frac{\delta H}{\delta \phi^{\prime \prime}(\mathbf{x}, t)},
$$




$$
\partial_{t} \phi^{S}(\mathbf{x}, t)=-\frac{\delta H}{\delta h(\mathbf{x}, t)}+Q\left(h(\mathbf{x}, t), \phi^{s}(\mathbf{x}, t)\right) .
$$

The functional $Q$ determines the rate of viscous dissipation in Eqs. (A5) and (A6) so that

$$
\frac{d l l}{d t}-\frac{\partial I l}{\partial t}=\int d \mathbf{x} Q\left(h(\mathbf{x}, t), \phi^{s}(\mathbf{x}, t)\right) \partial_{l} h(\mathbf{x}, t) .
$$

Of course, $Q=0$ corresponds to the inviscid limit.

The case of a fluid of low viscosity has been treated by assuming that energy dissipation is dominated by potential flow in the bulk [29]. The functional $Q$ can then be determined by equating the rate of dissipation in Eq. (A7) to the rate of energy dissipation due to potential flow,

$$
\begin{aligned}
& \int d \mathbf{x} Q(h(\mathbf{x}, t), \phi(\mathbf{x}, t)) \partial_{t} h(\mathbf{x}, t) \\
& =-v \int d \mathbf{x} \int_{\omega}^{h(\mathbf{x}, t)} d z \nabla^{2}(\nabla \phi)^{2} .
\end{aligned}
$$

This equation has been used to determine $Q$ order by order in an expansion in the surface wave steepness $[40,41]$. To the order relevant here, one finds,

$$
\hat{Q}(\mathbf{k}, t)=-4 \nu^{2} \hat{\phi}^{S}(\mathbf{k}, t)+(\text { nonlincar terms }),
$$

where $\hat{Q}(\mathbf{k}, t)$ is the Fourier transform of $Q$. As discussed in Refs. $[10,11]$, this approximation yields, by construction, the correct rate of energy dissipation at linear order, but not the correct equations of motion even at this order. In particular, it overestimates by a factor of 2 the damping force in Eq. (AG), and omits wave rectification in Eq. (A5) that arises from the rotational component of the flow in a thin boundary layer adjacent to the free surface.

Following Zakharov [38], we define a complex ficld

$$
b(\mathbf{k}, t)=\sqrt{\frac{\omega(k)}{2 k}} \hat{h}(\mathbf{k}, t)+i \sqrt{\frac{k}{2 \omega(k)}} \hat{\phi}^{s}(\mathbf{k}, t),
$$

where $\hat{h}(\mathbf{k}, t)$ and $\hat{\phi}(\mathbf{k}, t)$ are the two-dimensional Fourier transforms of $h(\mathbf{x}, t)$ and $\phi^{s}(\mathbf{x}, t)$, respectively, and $\omega(k)$ $=\sqrt{g_{0} k+\sigma k^{3} / \rho}$ is the inviscid dispersion relation. In terms of this new variable, the Hamiltonian system (A5) and (A6) can be written as

$$
\partial_{t} b(\mathbf{k}, t)=-i \frac{\delta H}{\delta \phi(-\mathbf{k}, t)}+i \sqrt{\frac{k}{2 \omega(k)}} \hat{Q}(\mathbf{k}, t) .
$$

Equation (A11) is now expanded in a power serics of $b$. We confine ourselves here to linear terms in $b$, as nonlinear terms will be added phenomenologically. However, explicil forms of cubic terms in $b$ have been obtained [42] both for the present case of an expansion around the inviscid solution, and also for the linear damping quasipotential equations of Ref. [11].

By expanding Eq. (A11) in power series of $b$, we find

$$
\begin{aligned}
\partial_{t} b(\mathbf{k}, t)+2 \nu k^{2}[b(\mathbf{k}, t)-\bar{b}(-\mathbf{k}, t)]+i \omega(k) b(\mathbf{k}, t) \\
\quad+\frac{i k g_{z}(t)}{2 \omega(k)}[b(\mathbf{k}, t)+\overline{b(}(\mathbf{k}, t)]+\mathcal{N} \mathcal{C}(b(\mathbf{k}, t))=0
\end{aligned}
$$

where $\mathcal{N} \mathcal{C}(b(\mathbf{k}, t))$ stands for terms nonlinear in the ampliludes $b$.

If the driving accelcration is given by $g_{3}(t)=a \cos \Omega t$, only amplitudes with wave number close to the critical wave number $k_{0}$ are excited near onset, with frequency close to the resonant frequency $\omega\left(k_{0}\right)=\Omega / 2$. We introduce a conventional multiple scale expansion near onset, but choose to do in a manner that will preserve the rotational invariance of the original governing equations. We further assume the following scalings for the damping and driving terms: $\gamma^{\prime}=2 \nu k_{i 1}^{2}$ $=\epsilon^{2} \gamma_{0}$ and $f^{t}=k_{0} a /\left[4 \omega\left(k_{0}\right)\right]=\epsilon^{2} f_{0}$, where $\epsilon$ is a small expansion parameter, and both $\gamma_{0}$ and $f_{0}$ are $O(1)$ quantities. We also expand

$$
b(\mathbf{k}, t)=\boldsymbol{\epsilon} B\left(\mathbf{k}, T_{1}, T_{2}\right) e^{\left.-i \omega_{i} k_{0}\right) t}+\epsilon^{2} b_{2}(\mathbf{k}, t)+\boldsymbol{\epsilon}^{3} b_{3}(\mathbf{k}, t)+\cdots
$$

with $T_{1}=\epsilon t$ and $T_{2}=\epsilon^{2} t$. The slow time scale $T_{1}$ corresponds to the time scale of translation of a wave packet, whereas $T_{2}$ is the scale of change in the modulation of the wave packet. These two time scales are consistent with an expansion of the inviscid dispersion relation $\omega(k)=\omega\left(k_{0}\right)+\epsilon \omega^{\prime}+\epsilon^{2} \omega^{\prime \prime}$ $+\cdots$ for modes near the critical wave number $k_{0}$. Substitution of Eq. (A13) into Eq. (A12) shows that the equation is identically satisfied at $O(\epsilon)$. At $O\left(\epsilon^{2}\right)$, we obtain the following solvability condition:

$$
\frac{\partial B}{\partial T_{1}^{\prime}}=-i \omega^{\prime} B
$$

The solvability condition at order $O\left(\epsilon^{3}\right)$ is

$$
\frac{\partial B}{\partial T_{2}}=-\gamma_{0} B(\mathbf{k})-i f_{i} B(-\mathbf{k})-i \omega^{\prime \prime} B(\mathbf{k})+\mathcal{N} C[B]
$$

with a known nonlinear functional $\mathcal{N C}[B]$. We now combine the two solvability conditions by writing $A(\mathbf{k})=\epsilon B(\mathbf{k})$ and $\partial_{t} A=\epsilon^{2} \partial_{T_{1}} B+\epsilon^{3} \partial_{T_{2}} \beta$ and find

$$
\begin{aligned}
\frac{\partial A}{\partial t}= & -\gamma^{\prime} A(\mathbf{k}, t)-i f^{\prime} \bar{A}(-\mathbf{k}, t)-i\left(\epsilon \omega^{\prime}+\epsilon^{2}(t)^{\prime \prime}\right) A(\mathbf{k}, t) \\
& +\mathcal{N C}[A] .
\end{aligned}
$$

Hence the slow evolution near onset given by Eq. (A16) is the same as that of the original set of inviscid equations supplemented by phenomenological linear damping.

From the inviscid dispersion relation, we find

$$
\epsilon()^{\prime}+\epsilon^{2} \omega^{\prime \prime}=c_{1}\left(k^{2}-k_{0}^{2}\right)+c_{2}\left(k^{2}-k_{0}^{2}\right)^{2}+O\left(\left(k-k_{0}\right)^{3}\right),
$$

with 


$$
c_{1}=\frac{g_{0}}{4 k_{0} \omega\left(k_{0}\right)}+\frac{3 \sigma k_{i}}{4 \rho \omega\left(k_{0}\right)}
$$

and

$$
c_{2}=\frac{1}{4 k_{0}^{2} \omega\left(k_{0}\right)}\left(\frac{3 \sigma k_{0}}{4 \rho}-\frac{\left(g_{0}+3 \sigma k_{0}^{2} / \rho\right)^{2}}{8 \omega\left(k_{0}\right)^{2}}-\frac{g_{0}}{4 k_{i 0}}\right) .
$$

We derive next a real-space order parameter model from Eq. (A16). Three simplifications are necessary. First the nonlinear functional in Eq. (A16) does not have a closedform representation in real space. As has been done in other systems (cf. Rayleigh-Bénard convection [43]), we introduce phenomenological functional forms for this term. In doing so, we artificially determine the symmetry of the bifurcating pattern at onset, but more importantly in the case of Faraday waves, we sidestep the issue of the origin of nonlinear damping and saturation of the waves $[11,44]$. In the simplest possible case, the nonlinear term in Eq. (A16) is approximated by an imaginary constant $i R^{\prime}$. Second, it is also known that lincar damping is not sufficient to produce wave saturation in this system [40]. We introduce a phenomenological nonlinear damping coefficient $\alpha \gamma^{\prime}$, where $\alpha$ is a constant assumed to be of order 1 .

We finally define a complex order parameter field $\psi(\mathbf{x}, t)$ as the inverse Fourier transform of $A(\mathbf{k})$, and find from Eq. (A16),

$$
\begin{aligned}
\partial_{t} \psi= & -\gamma^{\prime} \psi+i f^{\prime} \psi+i c_{1}\left(k_{0}^{2}+\nabla^{2}\right) \psi-i c_{2}\left(\nabla^{2}+k_{0}^{2}\right)^{2} \psi \\
& +\left(-\alpha \gamma^{\prime}+i R^{\prime}\right)\|\psi\|^{2} \psi .
\end{aligned}
$$

We now choose $\omega\left(k_{0}\right)=\Omega / 2$ as the unit of time, $1 / k_{0}$ as the unit of length, and further define $f=f^{\prime} / \omega\left(k_{0}\right)=k_{0} a / 4 \omega\left(k_{0}\right)^{2}$, $\gamma=\gamma^{\prime} / \omega\left(k_{0}\right)=2 \nu k_{0}^{2} / \omega\left(k_{0}\right)$, and $R=R^{\prime} / \omega\left(k_{0}\right)$. By choosing $1 / \sqrt{ } R$ as the order parameter scale, Eq. (113) results in the capillary wave limit. The positive sign of the imaginary part of the nonlinear coefficient $(-\alpha \gamma+i)$ in Eq. (113) is chosen to represent capillary waves. In the opposite limit of gravity waves, the imaginary part of this coefficient has to be negative. Note that as a third simplification we have eliminated the term $i\left(\mathbf{l}+\nabla^{2}\right)^{2} \psi$ in Eq. (113), as this term together with $i\left(\mathbf{1}+\boldsymbol{\nabla}^{2}\right) \psi$ leads to two different wave numbers becoming critical at threshold, an unwanted feature for us.

The effect of this third simplification can be further understood by comparing the amplitude equation on the model and that of the inviscid fluid. By introducing a multiple scale expansion of the form

$$
\psi=\delta \sum_{j- \pm 1}^{+N} a_{j}\left(\mathbf{X}, t, T_{1}, T_{2}\right) e^{j \hat{\mathbf{k}_{j}} \mathbf{x}}+\delta^{2} \psi_{2}+\delta^{3} \psi_{3} \cdots
$$

with $\delta$ a small bookkeeping parameter, and $\mathbf{X}=\delta \mathbf{x}, T_{1}=\delta t$, and $T_{2}=\delta^{2} t$, we find that up to order $O\left(\delta^{3}\right)$ [10]

$$
\begin{aligned}
\partial_{i} a_{j}= & -\gamma a_{j}+i f \bar{a}_{j}-\frac{3}{2}\left(\hat{\mathbf{k}}_{j} \cdot \boldsymbol{\nabla}\right) a_{j}+\frac{3 i}{4} \nabla^{2} a_{j}+(-\alpha \gamma+i) \\
& \times\left(\left|a_{j}\right|^{2} a_{j}+2 \sum_{l / j}\left|a_{i}\right|^{2} a_{j}+2 \sum_{i / \perp_{j}} a_{l} a_{-l} \bar{a}_{-j}\right)
\end{aligned}
$$

by following the same expansion procedure outlined above. The terms linear in the amplitudes are the same as the corresponding terms in the amplitude equation derived directly from the inviscid equations except for an additional term $\left(\hat{\mathbf{k}}_{j} \cdot \boldsymbol{\nabla}\right)^{2} a_{j}$, which is missing in Eq. (A22). This is a direct consequence of having eliminated the term $i\left(1+\nabla^{2}\right)^{2} / s$ in the phenomenological model.
[1] M. Faraday, Philos. Trans. R. Soc. London 121, 319 (1831).

[2] J. Miles and D. Henderson, Annu. Rev. Fluid Mech. 22, 143 (1990)

[3] M. Cross and P. Hohenberg, Rev. Mod. Phys. 65, 851 (1993).

[4] J. Gollub and J. Langer, Rev. Mod. Phys. 71, S396 (1999).

[5] A. Kudrolli and J. Gollub, Physica D 97, 133 (1996).

[6] 1). l3inks and $W$. van de Water, Phys. Rev. l.ett. 78, 4043 (1997).

[7] D. Binks, M.-T. Westra, and W. van de Water, Phys. Rev. Lett. 79, 5010 (1997).

[8] M.-T. Westra, D. Binks, and W. van de Water, J. Fluid Mech. 496, 1 (2003).

[9] W. Edwards and S. Fauve, J. Fluid Mech. 278, 123 (1994).

[10] W. Zhang, Ph.D. thesis, Florida State University (1994).

[11] W. Zhang and J. Viñals, J. Fluid Mech. 336, 301 (1997).

[12] V. Lapuerta, C. Martel, and J. M. Vega, Physica D 173, 178 (2002).

[13] J. M. Vega, E. Knobloch, and C. Marlel, Physica D 154, 313 (2001).

[14] M. I Iiguera, J. M. Vega, and I.. Knobloch, in Coherent Siructures in Complex Sysiems, edited by 1.. I3onilla, G. Platero, 1). Reguera, and J. Rubi (Springer-Verlag, New York, 2001), p.
328.

[15] M. Higucra, J. M. Vega, and E. Knobloch, J. Nonlinear Sci. $12,505(2002)$.

[16] A. Liu and S. Davis, J. Fluid Mech. 81, 63 (1977).

[17] S. Leibovich and S. Paolucci, J. Fluid Mech. 102, 141 (1981).

[18] A. Craik, Wave Interactions and Fluid Flows (Cambridge University Press, Cambridge, 1985).

[19] M. Iskandarani and P. Liu, J. Fluid Mech. 231, 417 (1991).

[20] P. Milewsky and D. Benney, Stud. $\Lambda$ ppl. Math. 94, 131 (1995).

[21] F. Mashayck and N. Ashgriz, Phys. Fluids 10, 1071 (1998).

[22] $\Lambda$. Davey and S. Stewartson, Proc. R. Soc. London, Ser. $\Lambda$ 338, 101 (1974).

[23] M. Longuet-Higgins, Philos. Trans. R. Soc. London, Scr. $A$ 245, 535 (1953).

[24] W. Zhang and J. Viñals, Physica D 116, 225 (1998).

[25] M. Uneki, Phys. Rev. Lel1. 67, 2650 (2002).

[26] Z. Feng and S. Wiggins, Nonlinear Dyn. 67, 141 (1995).

[27] H. Arel and C. Martel, Phys. Fluids 14, 1315 (2002).

[28] (i. Batchelor, An Iniroducion io liluid Dynamics (Cambridge University Press, Cambridge, 1967).

[29] I.. l.andau and 1:. l.ifshitz, Mechanics (Pergamon, New York, 
1976).

[30] J. Nicolás and J. M. Vega, liluid I)yn. Res. 32, 119 (2003).

[31] P. L.-F. Liu, Coastal Eng. 17, 207 (1977).

[32] P. Chen and J. Viñals, Phys. Rev. E 60, 559 (1999).

[33] W. Zhang and J. Viñals, Phys. Rev. Lett. 74, 690 (1995).

[34] S. Kiyashko, L. Korzinov, M. Rabinovich, and L. Tsimring, Phys. Rev. E 54, 5037 (1996).

[35] P. Chen and K. Wu, Phys. Rev. Lelt. 85, 3813 (2000).

[36] M. Golubitsky, I. Stewarl, and D. Schaefler, Singularities and Groups in Bifurcation Theory, Vol. II (Springer, New York, 1988).
[37] J. Crawford, Phys. Rev. lett. 67, 441 (1991).

[38] V. /akharov, //h. Prikl. Mekh. Tekh. liiz. 9.86 (1968) [J. Appl. Mech. Tech. Phys. 9, 190 (1968)].

[39] D. Crawford, P. Saffman, and H. Yuen, Wave Motion 2, 1 (1980).

[40] S. Milner, J. Fluid Mech. 225, 81 (1991).

[41] P. Lyngshansen and P. Alstrom, J. Fluid Mech. 351, 301 (1997).

[42] W. Zhang and J. Viñals (unpublished).

[43] J. Swill and P. Hohenberg, Phys. Rev. A 15, 319 (1977).

[44] P. Chen and J. Viñals, Phys. Rev. Lett. 79, 2670 (1997). 